\title{
Measurement of $\Omega_{m}, \Omega_{\Lambda}$ from a blind analysis of Type Ia supernovae with CMAGIC: Using color information to verify the acceleration of the Universe
}

\author{
A. Conley ${ }^{1,2,8}$, G. Goldhaber ${ }^{1,2}$, L. Wang ${ }^{1}$, G. Aldering ${ }^{1}$, R. Amanullah ${ }^{3}$, E. D. Commins ${ }^{2}$, \\ V. Fadeyev ${ }^{1}$, G. Folatelli ${ }^{4}$, G. Garavini ${ }^{5}$, R. Gibbons ${ }^{6}$, A. Goobar ${ }^{3}$, D. E. Groom ${ }^{1}$, I. Hook ${ }^{7}$, \\ D. A. Howell ${ }^{8}$, A. G. Kim ${ }^{1}$, R. A. Knop ${ }^{6}$, M. Kowalski ${ }^{1}$, N. Kuznetsova ${ }^{1}$, C. $\operatorname{Lidman}^{9}$, S. Nobili ${ }^{5}$, \\ P. E. Nugent ${ }^{1}$, R. Pain ${ }^{5}$, S. Perlmutter ${ }^{1,2}$, E. Smith ${ }^{6}$, A. L. Spadafora ${ }^{1}$, V. Stanishev ${ }^{3}$, \\ M. Strovink ${ }^{1,2}$, R. C. Thomas ${ }^{1}$, and W. M. Wood-Vasey ${ }^{1,2}$ \\ (THE SUPERNOVA COSMOLOGY PROJECT) \\ conley@astro.utoronto.ca
}

\begin{abstract}
We present measurements of $\Omega_{m}$ and $\Omega_{\Lambda}$ from a blind analysis of 21 high-redshift supernovae using a new technique (CMAGIC) for fitting the multi-color light-curves of Type Ia supernovae, first introduced by Wang et al. (2003). CMAGIC takes advantage of the remarkably simple behavior of Type Ia supernovae on color-magnitude diagrams, and has several advantages over current techniques based on maximum magnitudes. Among these are a reduced sensitivity to host galaxy dust extinction, a shallower luminosity-width relation, and the relative simplicity of the fitting procedure. This allows us to provide a cross-check of previous supernova cosmology results, despite the fact that current data sets were not observed in a manner optimized for CMAGIC. We describe the details of our novel blindness procedure, which is designed to prevent experimenter bias. The data are broadly consistent with the picture of an accelerating Universe, and agree with a flat Universe within $1.7 \sigma$, including systematics. We also compare the CMAGIC results directly with those of maximum magnitude fits to the
\end{abstract}

\footnotetext{
${ }^{1}$ E. O. Lawrence Berkeley National Laboratory, 1 Cyclotron Rd., Berkeley, CA 94720, USA

${ }^{2}$ Department of Physics, University of California Berkeley, Berkeley, 94720-7300 CA, USA

${ }^{3}$ Department of Physics, Stockholm University, Albanova University Center, S-106 91 Stockholm, Sweden

${ }^{4}$ Observatories of the Carnegie Institution of Washington, 813 Santa Barbara St., Pasadena, CA 91101

${ }^{5}$ LPNHE, CNRS-IN2P3, University of Paris VI \& VII, Paris, France

${ }^{6}$ Department of Physics and Astronomy, Vanderbilt University, Nashville, TN 37240, USA

${ }^{7}$ Department of Physics, University of Oxford, Nuclear \& Astrophysics Laboratory, Keble Road, Oxford, OX1

${ }^{8}$ Department of Astronomy and Astrophysics, University of Toronto, 50 St. George St., Toronto, Ontario M5S 3H4, Canada

${ }^{9}$ European Southern Observatory, Alonso de Cordova 3107, Vitacura, Casilla 19001, Santiago 19, Chile
} $3 \mathrm{RH}, \mathrm{UK}$ 
same supernovae, finding that CMAGIC favors more acceleration at the $1.6 \sigma$ level, including systematics and the correlation between the two measurements. A fit for $w$ assuming a flat Universe yields a value that is consistent with a cosmological constant within $1.2 \sigma$.

Subject headings: cosmological parameters — cosmology: observations — supernovae: general

\section{INTRODUCTION}

Type Ia supernovae (SNe Ia) have proved to be an extremely valuable tool for measuring the cosmological parameters, as they are the best high-luminosity standard candles currently known to astronomy. Studies of the peak $B$-band luminosities of high redshift SNe Ia led to the surprising discovery by two independent groups (the Supernova Cosmology Project (SCP; Perlmutter et al. 1998, Perlmutter et al. 1999 (hereafter P99)) and the High-z Supernova Search Team (HZSST; Garnavich et al. 1998; Schmidt et al. 1998; Riess et al. 1998), that the expansion of the Universe is accelerating. This acceleration is consistent with some form of 'dark energy', possibly Einstein's cosmological constant $\Lambda$. The implications of this result for the future fate of the Universe and our understanding of fundamental physics are profound; therefore, it is extremely important that it be verified by independent methods.

The best approach is to make use of alternative measurements that depend on other physical processes. There are now several additional lines of evidence that support the accelerating Universe, but most are based on combining several different measurements. For example, the combination of the angular size of fluctuations on the surface of last scattering of the cosmic microwave background (CMB) with measurements of the clustering of mass on large scales (Spergel et al. 2003; Tegmark et al. 2004; Eisenstein et al. 2005) provides strong evidence for a dark energy component. There is also a direct detection of dark energy using the integrated Sachs-Wolfe effect (Padmanabhan et al. 2005). It is encouraging that these different lines of evidence, which depend on very disparate physical processes and probe very different cosmic epochs, are consistent with a $\Omega_{m} \sim 0.3, \Omega_{\Lambda} \sim 0.7$ Universe.

Still, SNe Ia provide the best direct evidence for dark energy, and any improvement in our understanding of their properties is very welcome. There are several possible alternative explanations for the SN result. Since dark energy manifests itself in this context as high-redshift SNe Ia being slightly dimmer than expected, the most obvious alternative explanation is that this dimming is caused by extragalactic dust, either in intergalactic space or in the host galaxies of the SNe. Another possibility, and a significantly more difficult one to quantify, is that high redshift $\mathrm{SNe}$ are somehow dissimilar from low redshift SNe in a way that we have not yet detected. This paper presents results based on an analysis of SNe Ia with a new method (CMAGIC, for ColorMAGnitude Intercept Calibration) introduced in Wang et al. (2003) (hereafter W03) that partially 
addresses both issues.

There is no unique choice for the magnitude to associate with an SN Ia because their luminosity varies in time. For convenience, virtually all previous studies have used the $B$ magnitude at maximum brightness, $m_{B}$, as the standardized candle, but there is no a priori reason why this choice is optimal. $m_{B}$ is generally determined by fitting an empirical curve to the $B$-band brightness as a function of time and reading off the peak value. When available, observations in other passbands are frequently incorporated into the fitting procedure. There is a well-established empirical relation between absolute magnitude and the width of the light curve as parameterized by stretch (Perlmutter et al. 1997; P99; Goldhaber et al. 2001), $\Delta m_{15}(B)$ (Phillips 1993; Phillips et al. 1999) or the MLCS parameter $\Delta$ (Riess et al. 1996) in the sense that SNe with wider, more slowly declining light curves (high stretches) are intrinsically brighter. Here the stretch parameterization is used.

Since ordinary interstellar dust both extinguishes and reddens light, P99 compared the distributions of $B-V$ colors at maximum luminosity of the low and high redshift SN samples, finding no significant evidence that the high redshift sample is more reddened. It should be emphasized that the SN measurement of $\Omega_{m}$ and $\Omega_{\Lambda}$ is relative - as long as the low- and high-redshift samples suffer the same amount of extinction (or any other bias), there is no effect on the final result. Sullivan et al. (2002) decomposed the SN sample into subsets based on the Hubble type of their host galaxies, a powerful approach because early-type galaxies are expected to have little or no dust, and found that $\Omega_{\Lambda}$ was detected in each subsample. A difficulty with this analysis is that the resulting error bars on $\Omega_{m}, \Omega_{\Lambda}$ are necessarily much larger because the morphological subsets have considerably fewer SNe than the full sample.

One may attempt to measure the reddening for each SN by measuring its color and correcting for host galaxy extinction by assuming a dust extinction law. The error in the extinction correction usually dominates the statistical errors of each SN. In early work the HZSST team made use of an asymmetric prior on the intrinsic extinction distribution to limit the propagated uncertainties resulting from the extinction correction (Riess et al. 1998) while performing light-curve fits, which can bias the results under some circumstances (P99). More recent papers have made improvements in the form of the prior and its application and corrected this problem (Barris et al. 2004; Riess et al. 2004), although at the potential cost of enhanced sensitivity to any evolution in the extinction distribution. Knop et al. (2003) (hereafter K03) made use of high quality color measurements made possible by the Hubble Space Telescope (HST) to estimate the extinction values of individual SN without making use of such a prior.

The evolution issue is extremely difficult to address. To first order evolution should not be a concern because the diversity of the environments in which local SNe Ia occur is much larger than the mean difference in environment between the high and low redshift samples. While there are some properties of SNe Ia that are known to correlate with host environment, these correlations disappear once the width-luminosity relation is taken into account (Hamuv et al. 2000). The analysis of Sullivan et al. (2002) also has relevance for this question because it compares SNe Ia from similar 
host environments at high and low redshift. One can also compare individual SNe in more detail spectroscopically (Hook et al. 2005), although such measurements are taxing even for modern 8-10m class telescopes. In a spectroscopic study of 12 high redshift SNe, Garavini et al. (2005) found no evidence for evolution.

CMAGIC offers some benefits with respect to dust and evolutionary models, as described further in 2.2 and 2.3 . It is possible to define a standard candle magnitude with CMAGIC, and because of the nature of the CMAGIC relationships, this magnitude is affected by the same amount of dust by roughly half as much as $m_{B}$. On the evolutionary front the situation is more complicated. There are some potential evolutionary effects for which CMAGIC offers advantages, but it is uncertain how important this is because the effects of these theories have not been clearly delineated. Because CMAGIC depends on light-curve data in a very different fashion than maximum magnitude fits, and in particular because it is much more sensitive to later epochs relative to maximum light, for some potential evolutionary effects we can expect the CMAGIC magnitude to be affected differently. However, this is difficult to quantify given the current lack of detailed predictions from theories of SN evolution. Combining these two considerations, CMAGIC can provide a powerful cross check of previous SNe Ia cosmology results. Because we are attempting to verify previous results, it is important to prevent the analysis from being unintentionally biased towards the expected outcome. To this end a blindness technique has been developed and used during the cosmological analysis in this paper.

Perhaps for some of the above reasons, low redshift SNe Ia analyzed with CMAGIC have a smaller intrinsic variation than the maximum magnitudes of the same SNe without extinction correction $\left(\sigma_{\text {int }}=0.12 \mathrm{mag}\right.$, compared with approximately $0.17 \mathrm{mag}$ for $\left.m_{B}\right)$. For many current data sets, the intrinsic variation dominates over observational errors, so CMAGIC may allow us to obtain tighter constraints on the cosmological parameters for a similar observational expense in future surveys.

The goals of this paper are twofold: (1) to show that the CMAGIC relations hold at high redshift for well measured $\mathrm{SNe}$, and (2) to measure the cosmological parameters from already existing data sets and use this to cross-check previous results. We first describe CMAGIC in more detail (\$2). We then describe the data sample (\$3) and the CMAGIC fitting procedures (\$4), and then we use these to demonstrate that CMAGIC works for high redshift SNe (55). Once this is established, we proceed to the primary analysis of this paper, the cosmological fits. First we describe the cosmological fitting techniques ( 96 ), including a discussion of the blindness technique (\$6.3). Finally, the cosmological results are presented (\$7), systematic effects are discussed (\$8), and the results are analyzed (99). 


\section{CMAGIC}

CMAGIC is described in considerably more depth in W03. Here we provide a brief review of the relations, define the magnitude $\left(B_{B V 0.6}\right)$ used in this study, and discuss the benefits of CMAGIC with respect to extinction and evolution.

\subsection{CMAGIC Relations}

CMAGIC is based on the behavior of SNe Ia in color-magnitude diagrams. Starting approximately 1 week after $B$ maximum and lasting approximately 3 weeks, the relation between the $B$ magnitude and $B-V$ color is strikingly linear. This holds true for other colors as well (at least $B-R, B-I)$. Some typical low redshift examples are shown in figure 1 The temporal extent of this linear region is a function of stretch, with slower, higher stretch light-curves starting and ending their linear behavior later. The slope, $\beta$, of the linear region has a narrow distribution. Currently very few rest-frame $R$ and $I$ observations are available for high redshift SNe Ia, so here we consider only $B$ versus $B-V$. The simplicity of this behavior is so far not completely explained by theory, which gives it a status similar to the empirical width-luminosity relation. Prior to the linear region, the majority of SNe Ia are less luminous than the linear extrapolation. However, a minority (typically those with high stretch) display excess luminosity, which is referred to as a 'bump'. Standard light-curve template fitting techniques (stretch, MLCS) do not adequately reproduce the CMAGIC relations. Both issues are discussed in more detail in W03.

The distribution of slopes in this linear region is fairly narrow, with $\left\langle\beta_{B V}\right\rangle=1.98$ and a RMS of 0.16, as shown in figure 2 for low-redshift SNe Ia. To first order, $\beta_{B V}$ is affected by $K$-corrections but not by extinction. W03 explored fixing the slope at the mean value for all fits. The effects of this assumption are quite minor, but it is possible to improve on this procedure by including information about the distribution of slopes in the fitting procedure (\$4).

The CMAGIC relation for $B$ versus $B-V$ can be written conveniently in the form

$$
B=B_{B V 0.6}+\beta_{B V}(B-V-0.6),
$$

which defines $B_{B V 0.6}$ as the $B$ magnitude when $B-V=0.6$; this is the magnitude used as a standard candle in this paper. The particular $B-V$ color is chosen to minimize the covariance between the standard candle magnitude and the slope $\beta_{B V}$, as it is approximately the mean $B-V$ color in the linear region of an unextinguished SN Ia. Because the color roughly measures the ejecta temperature, by evaluating the magnitude at a fixed color we essentially ensure that all $\mathrm{SNe}$ are evaluated at a point where their physical properties are similar.

The behavior of an SN Ia on a CMAGIC diagram can also be viewed temporally. Proceeding in a clockwise fashion around the curves in figure 1. a typical, unextinguished SN Ia usually has a color of approximately $B-V=0$ at maximum, and evolves rapidly to the red for about a month. 
After this it enters the so-called nebular phase and evolves bluewards, again in a linear fashion. This second linear region has some interesting properties, but since data at such late epochs are very rarely available for high-redshift $\mathrm{SNe}$, we do not discuss it further here. With good time coverage it is possible to determine the extent of the linear region by examination, but this is generally not possible with current high redshift data. Fortunately, the beginning and ending dates of the linear region relative to the date of $B$ maximum form a well-defined sequence in terms of stretch and the presence or absence of the bump feature. Using well-observed low-redshift SNe to determine the earliest and latest points in the linear region as a function of stretch, we find that the beginning date of the linear region is well described by $t_{b}=5+3(s-1)$ and the ending date by $t_{e}=29+40(s-1)$, where both are measured in rest-frame days relative to $B$ maximum and $s$ is the stretch. SNe Ia with bumps (e.g., the lower panel of figure 1) do not fit smoothly into this scheme and are well represented by $t_{b}=13.5$ and $t_{e}=30$. This suggests a possible source of bias in the analysis of the high redshift sample, since the presence or absence of a bump may be difficult to detect given the typical quality of high redshift photometry. Fortunately, for this data sample this issue proves to be unimportant (Appendix $\mathrm{A}$ ).

Detailed studies (Appendix $\mathrm{B}$ ) show that the fitting procedure induces weak negative correlations between $B_{B V 0.6}$ and $m_{B}$, at least for current light-curve templates. Clearly, these templates have missed some aspect of SNe Ia behavior (or the correlations would be much stronger), and $B_{B V 0.6}$ provides some additional information that can be used to constrain the cosmological parameters. Peculiar velocities, stretch correction, and extinction induce additional correlations between these magnitudes.

\subsection{Host Galaxy Dust}

Interstellar dust is a major component of our and other galaxies. A good review can be found in Draine (2003). Ordinary dust both extinguishes and reddens starlight because it absorbs blue light more strongly than red light. The relative amount of absorption between wavelengths is characterized by an absorption law such as that of Cardelli et al. (1989). For an object with a stellar spectrum, the extinction in the $B$-band $A_{B}$ (in magnitudes) is related to the amount of reddening $E(B-V)$ by $A_{B}=\mathcal{R}_{B} E(B-V)$. For SNe, which do not have stellar-like spectra, and whose spectral features change with time, this is not strictly appropriate, but $\mathcal{R}_{B}$ is still useful as a parameterization of the extinction law. A typical value in our Galaxy is $\mathcal{R}_{B}=4.1$, although it varies considerably along different lines of sight (Fitzpatrick 1999). The characteristic scatter of $\mathcal{R}_{B}$ is not well constrained.

So far it has not been feasible to measure the extinction law directly for the host galaxies of high redshift $\mathrm{SNe}$, so the general approach has been to assume that the $\mathcal{R}_{B}$ values for the high and low redshift SNe samples are identical. This assumption takes several forms. In the primary fit of P99 (fit C) no extinction correction is performed, but it is argued that the similarity of the observed $E(B-V)$ distributions of the two samples implies that host galaxy dust extinction is 
not contaminating the cosmological results. Because $\mathcal{R}_{B}$ is necessary to transform $E(B-V)$ into the amount of extinction, this is tantamount to assuming that $\mathcal{R}_{B}$ is the same for the two samples. There is a theoretical and empirical expectation that the SN sample suffers from relatively little extinction (Hatano et al. 1998). K03 perform an extinction correction by comparing the measured $B-V$ at maximum to an empirical model, then converting this to $A_{B}$ by assuming a value for $\mathcal{R}_{B}$. Riess et al. (1998, 2004); Tonrv et al. (2003); Barris et al. (2004) use a similar procedure. Previous analyses have generally performed a color cut on their SN samples on the theory that large color excesses may represent $\mathrm{SNe}$ in dustier environments where the value of $\mathcal{R}_{B}$ is likely to depart from the fiducial value. It is interesting to note that we may now have evidence for higher mean extinction at high redshift. The recent SN sample of Riess et al. (2004), which represents the deepest, highest redshift SN survey yet published, has much higher host galaxy extinction values than any other available SN sample, although survey selection effects may explain this result.

Because of the nature of the linear CMAGIC relations, the effective $\mathcal{R}$-value for $B_{B V 0.6}$ is approximately half of the value that it takes for $m_{B}$ (assuming a standard dust law), as shown schematically in figure 3. The critical point is that the magnitude is always evaluated at the same fixed color, and therefore the extinction and reddening effects partially cancel. Since SNe Ia redden as they evolve along the linear relation, $\mathcal{R}_{B_{B V 0.6}}=\mathcal{R}_{B}-\beta_{B V}$. For normal dust, $B_{B V 0.6}$ is less affected than $m_{B}$, which results in smaller uncertainties arising from the extinction correction, if a fixed $\mathcal{R}_{B}$ is assumed. Because the boundaries of the linear region are determined by date relative to maximum and not color, $B_{B V 0.6}$ remains less affected even if the amount of extinction is large enough that $B-V=0.6$ does not lie within the linear region. The precise epoch of maximum light is not nearly as important for $B_{B V 0.6}$ as it is for $m_{B}$ because the 'roll-off' at the edges of the linear region is much less severe than it is near peak luminosity. Note that CMAGIC offers no benefits with respect to an evolving $\mathcal{R}_{B}$ - the derivatives of $m_{B}$ and $B_{B V 0.6}$ with respect to $\mathcal{R}_{B}$ are identical. Nor does it offer any advantages for the so-called 'gray dust' $\left(\mathcal{R}_{B}=\infty\right)$ suggested by Aguierre (1999). Constraints on gray dust have been explored by Riess et al. (2000, 2004), but also see Nobili et al. (2003, 2005).

Since $B_{B V 0.6}$ and $m_{B}$ are affected by extinction differently, it is possible to estimate the amount of extinction by comparing the two magnitudes using the quantity $\mathcal{E}$, which is an estimator of $E(B-V)$ :

$$
\mathcal{E}=\frac{m_{B}-B_{B V 0.6}}{\beta_{B V}}+\text { const. }
$$

Using this correction substantially increases the correlations between $m_{B}$ and $B_{B V 0.6}$. Assuming a standard extinction law $\left(\mathcal{R}_{B}=4.1\right)$, the correlation coefficient between these two magnitudes climbs to $\rho>0.7$ from $\langle\rho\rangle=0.15$ (Appendix $(\mathbf{B}$ ), even in the absence of significant extinction. For this reason, this approach is not followed here. However, for smaller values of $\mathcal{R}_{B}$, such as those found by Tripp and Branch (1999) and Guv et al. (2005), this correlation is significantly reduced. 


\subsection{Evolution of SNe Ia}

The possibility that the average properties of SNe Ia have evolved between the current epoch and a redshift of 1 is of considerable concern for SN cosmologists. So far it has been impossible to demonstrate conclusively that evolution is not the cause of the claimed cosmological results. The best that can be done is to continue to quantitatively add "to the list of ways in which they are similar while failing to discern any way in which they are different" (Riess et al. 1999b). One method to approach this problem is to compare high and low redshift SNe in similar environments, as in Sullivan et al. (2002), where we found no evidence for evolutionary biases. Since all measured dependencies of SN Ia properties on local environment disappear after stretch correction, and because of the diversity of environments in which local SNe Ia occur, concerns about evolution can be usefully restricted to mechanisms that affect the width-luminosity relationship.

There are several theoretical models that predict possible avenues for evolution. Domínguez et al. (2001) and Höflich et al. (2000) have investigated the effects of decreasing metallicity and changing progenitor mass on SN Ia properties by constructing models of the progenitor star and then following them through detonation. If $\Delta$ is the change in $B-V$ they find that decreasing metallicity causes an SN to become slightly bluer $(\Delta=-0.05$ for an extreme case) without affecting the maximum $B$ magnitude. Most extinction corrections compare observed colors to empirically derived color relations to calculate the amount of extinction. If the intrinsic colors change, then the extinction correction will be incorrect. If no extinction correction is applied, then $m_{B}$ is unaffected, while $B_{B V 0.6}$ is overestimated by $\beta_{B V} \Delta \sim 2 \Delta$. If an extinction correction is applied, then for positive values of $\Delta$, the extinction correction for $m_{B}$ is overestimated and the SN is assigned an extinction-corrected magnitude that is too bright by $\mathcal{R}_{B} \Delta \sim 4 \Delta$. $\mathcal{E}$, by contrast, is underestimated, so once this correction is applied, $B_{B V 0.6}$ is too $\operatorname{dim}$ by $\beta_{B V} \Delta-\left(\mathcal{R}_{B}-\beta\right)^{2} \Delta / \beta_{B V}$. For typical values of $\beta_{B V}$ and $\mathcal{R}_{B}$, this cancels, and the extinction corrected value of $B_{B V 0.6}$ is unaffected by this evolutionary effect. In other words, either with or without extinction correction this particular evolutionary model will have different effects on $m_{B}$ and $B_{B V 0.6}$, so by comparing the two magnitudes this model can be evaluated against data. We note that the range of metallicities considered in this study is far greater than the expected change out to $z \sim 1$.

\section{DATA}

Currently available SN data sets have not been observed in a manner optimized for CMAGIC, particularly at high redshift. Out of the roughly $100 \mathrm{SNe}$ at $z>0.1$ with light curves available in the literature, only approximately 20 are useful for CMAGIC purposes. High redshift SNe are frequently not observed in the rest-frame $V$. Even when such observations do exist, they are usually only intended to establish the color at maximum for the purposes of applying an extinction correction, and therefore are usually concentrated too close to the peak to lie within the CMAGIC linear region. Future high redshift data sets (SNLS (Astier et al. 2005), ESSENCE (Matheson et al. 
2005), SDSS Supernova Search (Sako et al. 2005), SNAP (Aldering et al. 2004), LSST (Pinto et al. 2004)) will not suffer from this limitation, as they are designed to obtain multi-color photometry for almost all observed epochs. The current situation is considerably better for low redshift data sets, as many of these SNe have excellent multi-color coverage. There is an observational cost associated with CMAGIC because the linear region is $\sim 1.2 \mathrm{mag}$ dimmer than at peak, so the photometric error bars are larger for the same observational effort. Whether or not this extra cost is outweighed by the benefits with respect to dust and/or evolution depends on the specifics of the survey design.

We have attempted to construct a data sample including all SNe Ia with published light curves. In order to eliminate SNe that cannot be useful for the purposes of this paper, we enforce the following requirements. First, an object must be at least plausibly an SN Ia based on either light-curve shape, spectroscopic ID, or host galaxy morphology. Second, it must have at least one rest-frame $B-V$ observation. For this purpose we require that the central wavelength of the redshifted $B$ - or $V$-band lie within one HWHM of the central wavelength of the observed filter, which improves the reliability of the $K$-corrections by limiting the amount of extrapolation. We also do not include observations taken with extremely wide filters, such as F110W and F160W NICMOS filters on HST. These filters are wide enough that for many of the redshift ranges of interest they overlap considerably with both $B$ and $V$ (and sometimes $R$ ), making it difficult to measure $B-V$ in a fashion that is not heavily influenced by the model used to calculate the $K$-corrections. Clearly it must be possible to use these data in some fashion for CMAGIC, but it will require extreme care. Observations in $B$ and $V$ are only combined to form $B-V$ if they are within 0.5 rest frame days of each other; the analysis is quite insensitive to this value.

This results in a sample of $131 \mathrm{SNe}$, of which one third are at redshifts greater than 0.3. Note that we have not yet required that the $B-V$ point lie in the CMAGIC linear region, since this depends on the measured value of the stretch and date of maximum, or that the SN lie in the Hubble flow. The high-redshift portion of the sample comes from a fairly diverse set of sources. There are 14 from P99, six from K03, two from Garnavich et al. (1998), one from Schmidt et al. (1998), five from Riess et al. (1998), four from Tonrv et al. (2003), 13 from Barris et al. (2004), and one from Riess et al. (2004). The low-redshift sample is even more diverse, but primarily comes from three sources: Hamuv et al. (1996), Riess et al. (1999a) and Jha et al. (2005). Source information is provided in tables 1 and 2 Once a reasonable series of cuts are applied to this sample (\$6.2), approximately half of the SNe remain and are used in the cosmological analysis.

\section{CMAGIC FITTING PROCEDURES}

In order to determine if an individual data point lies within the linear CMAGIC region for a particular SN it is necessary to know the stretch and the date of $B$ maximum, although not to a high degree of accuracy. These are determined by performing a template fit to the $B$ and $V$ light curves in a manner similar to P99 and K03. Briefly, light-curve fits are performed using a $\chi^{2}$ minimization procedure based on MINUIT (James \& Roos 1975) with both $K$-corrections and 
corrections for Milky Way dust extinction taken into account. The light-curve template is that of K03 (which uses the $B$ template of Goldhaber et al. (2001) but a different $V$ template). For the photometry from P99 and K03, the photometric correlation matrices were used in the light-curve fits. These reflect the correlations between different observations of the same SN induced by the subtraction of the final reference image(s). For the literature objects, where this information was not available, the observations are assumed to be uncorrelated. In order to prevent systematic errors arising from differences in fitting procedures, we have only included SNe that we can treat consistently, i.e. with our own light-curve fitting procedure and $K$-corrections.

The correlation of the bump feature with different $B$ and $V$ stretch values complicates matters. As explained in Appendix A. SNe Ia with bumps can be fitted by the standard stretch templates if the ratio between $B$ and $V$ stretch values is allowed to vary. In order to handle this situation, three light-curve fits were performed for each $\mathrm{SN}$ - joint $B$ and $V, B$ only, and $V$ only. In joint fits the dates of maximum and stretch values of the two filters are fixed relative to each other by the light-curve template. Except when a bump is visible in the CMAGIC diagram, the joint fit is used. The reduced detectability of the bump feature at high redshift due to reduced data quality is a concern that is further discussed in Appendix A.

$K$-corrections play a critical role in this procedure. At high redshift cross-filter corrections are necessary (Kim et al. 1996), but even at low redshift same-filter $K$-corrections are not insignificant. Erroneous $K$-corrections alter the slope of the CMAGIC linear region, unlike extinction. Those used in this paper are based on the prescription of Nugent et al. (2002) but with the time series of spectral templates and empirical stretch-color relation of K03. Milky Way extinction is included in this calculation using the dust map of Schlegel et al. (1998). Our approach naturally takes into account the non-stellar nature of SN spectra and their variation with epoch. Errors associated with the $K$-corrections are discussed in 98 , where we also discuss the effects of several other modifications to the fitting procedure described here.

Since the $K$-correction is a function of stretch and epoch, the light-curve fits must be performed in an iterative manner. On the first iteration the stretch is set to 1 and the date of maximum is set to the date of the brightest point. The combined Milky Way and $K$-corrections are calculated and the light curve is fitted, and the new stretch and date of maximum are used to calculate new corrections. This process is iterated until convergence. The majority of SNe converge within three iterations, but the maximum number allowed is 16 . Those SNe that do not converge within 16 iterations invariably have extremely poor light-curve coverage and are excluded from the sample. Because high-redshift SNe very rarely have data beyond day 30, in order to prevent a bias between high and low redshift SNe in the fitting procedure data between 30 and 200 rest-frame days of maximum are not included, a similar procedure to that followed in P99 and K03. Observations more than 200 days after maximum light are included because they provide final reference information useful for setting the amount of host galaxy light underlying the SN.

This data set contains observations in 14 filters. $B V R I$ filter curves were obtained from 
Bessell (1990). We reiterate the warning of Suntzeff et al. (1999) that these filter functions include a linear function of $\lambda$, which we have removed. The same is true of the redshifted $B$ and $V$ filters used for some observations by the $\operatorname{HZSST}(B 35, V 35, B 45, V 45)$, with filter curves given by Schmidt et al. (1998). Filter curves for the HST filters on WFPC2 and ACS were generated using synphot (Simon and Shaw 1996). There are two sets of ground-based $z$-band observations: those from Tonry et al. (2003), and the $z^{\prime}$ observations taken with SuprimeCam on the Subaru telescope presented in Barris et al. (2004). The Tonrv et al. (2003) $Z$-band response curve is as presented in that paper, and the SuprimeCam $z^{\prime}$ system response was provided by H. Furusawa (2004, private communication).

Once the date of maximum and stretch are measured, the points in the CMAGIC linear region can be determined and the linear relation fitted. Note that the CMAGIC fit is performed on the observed data points, not on the template fit used to determine the stretch and date of maximum. Again a $\chi^{2}$ minimization routine is used based on MINUIT that allows for errors in both $B$ and $B-V$. The narrowness of the CMAGIC slope distribution, as shown in figure 2 led W03 to suggest fitting all CMAGIC relations with a fixed slope set at the mean of this distribution. This is particularly important when working with high-redshift SNe because the observational error bars are sufficiently large that accurate slope measurements are difficult. We can make better use of the available data by assuming that low- and high-redshift SNe have similar $\beta_{B V}$ distributions, as determined by examining low-redshift SNe. This is similar to the approach followed by previous analyses based on maximum magnitudes, where light-curve templates developed from low-redshift $\mathrm{SNe}$ are used to fit high redshift data. This leaves only one parameter in the fit, $B_{B V 0.6}$. However, it is possible to test the assumption that the slope distributions are consistent with the handful of high-redshift SNe with sufficiently small observational errors (\$5).

We improve on the fixed slope assumption by numerically propagating the additional error due to the observed distribution of slopes using a Monte-Carlo style approach. The slope distribution is determined from the low-redshift SN sample, which for this purpose includes SNe Ia that are not in the Hubble flow. We take care to apply the same cuts, described in 6.2 , on this sample as we do on the sample used to directly determine the cosmological parameters, except for the redshift cut. This approach slightly overestimates the errors because the measured slope distribution includes observational errors, but in any case the net effect is quite small, inflating the errors on $B_{B V 0.6}$

by around 0.01-0.03 mag in quadrature without affecting the central values. In other words, the assumption of a fixed slope used in W03 works extremely well for current data sets, although we do include the additional error term in this analysis.

\section{CMAGIC RELATIONS AT HIGH REDSHIFT}

The first task in applying CMAGIC at high redshift is to determine if SNe Ia at high redshift follow the linear relations derived at low redshift. A brief examination of the CMAGIC diagrams shows that high-redshift SNe do obey linear relations between magnitude and color. However, in 
order to put this statement on a more quantitative footing, we investigate the consistency of the $\beta_{B V}$ distributions. Most high-redshift observations have sufficiently large error bars that they do not provide useful slope constraints. However, there are a handful of relatively well observed SNe Ia that can be used to investigate this question: SNe 1997ce, 1997cj, 1998aw, 1998ax, and 1998ba. The requirement for membership in this set is that there be at least three points in the CMAGIC linear region and that $\sigma_{\beta_{B V}}<0.5$. SN 1997ce is particularly interesting because it clearly displays a bump feature. Whatever physical mechanism causes the bump feature is still active at high redshift.

The best fit slopes for these SNe are tabulated in table 3 and the CMAGIC diagrams are plotted in figure 5 The $\chi^{2}$ values for these fits are improbably low, suggesting that the photometric errors have been overestimated, which is also true of the low redshift sample. The slopes are histogrammed in figure 6. The mean slope for the low redshift sample is $\left\langle\beta_{B V}\right\rangle=1.98 \pm 0.03$ and for the high redshift sample it is $\left\langle\beta_{B V}\right\rangle=1.96 \pm 0.11$, so there is no evidence for disagreement. A stronger statement requires more high quality multicolor observations of high redshift SNe Ia.

\section{COSMOLOGY FITTING PROCEDURES}

We now proceed to the primary purpose of this paper, the cosmological analysis. Here we describe our methodology for performing these fits. The results presented here differ from previous papers in several respects. First, we have attempted to formalize the procedure whereby individual SNe are rejected or accepted into the data sample to a greater extent than has been true previously. Second, we make use of a blind analysis procedure in order to prevent experimenter bias from affecting the results. To this end, the results of the cosmological analysis have been hidden from the authors until the cuts and fitting procedure were finalized.

\subsection{Determining the Cosmological Parameters}

The luminosity distance equation can be written (in magnitudes) as

$$
m=5 \log _{10}\left(\mathcal{D}_{L}\left(z, \Omega_{m}, \Omega_{\Lambda}\right)\right)+\mathcal{M}-\alpha(s-1)
$$

where $m$ is the observed magnitude, $s$ is the stretch, $\mathcal{M}$ is a combination of the Hubble constant $H_{0}$ and the absolute magnitude of an $\mathrm{SN} \mathrm{Ia}$, and $\mathcal{D}_{L}$ is the $H_{0}$ free luminosity distance given in Perlmutter et al. (1997). Because of the somewhat complicated nature of this parameter space, the most conservative approach to fitting this relation is to perform a grid search over the four fitting parameters $\left(\Omega_{m}, \Omega_{\Lambda}, \alpha, \mathcal{M}\right)$ and then marginalize over the two nuisance parameters $(\mathcal{M}, \alpha)$. This is the procedure used in P99 and K03. Because of the highly nonlinear nature of the problem and the large errors on the cosmological parameters, looking for the point where the $\chi^{2}$ has increased

by 2.3 over its minimum leads to an underestimate of the errors. A $\chi^{2}$ is calculated at each point on 
the grid, making use of equation 3 and converted into a relative probability $P \propto \exp \left(-\chi^{2} / 2\right)$. The probabilities are then normalized over the grid, and the nuisance dimensions are summed over. The parameter ranges explored are $\Omega_{m}=[0,3], \Omega_{\Lambda}=[-1,4], \mathcal{M}=[24.7,25.5],{ }^{1}$ and $\alpha=[-0.5,2.0]$. These ranges include more than $99.99 \%$ of the probability. ${ }^{2}$

We have also constructed fits to the equation of state parameter $w$. In order to reduce the computational complexity of this problem, these fits are restricted to the flat universe case. Here the four parameters are $\Omega_{m}, w, \mathcal{M}$, and $\alpha$. $\mathcal{D}_{L}$ must be modified appropriately, but in all other respects the fit procedure is identical. The range of $w$ considered is $[0,-3.5]$.

The errors on each $B_{B V 0.6}$ include the following terms:

- The uncertainty from the CMAGIC fits, including a contribution from the distribution of $\beta_{B V}$.

- The uncertainty of the stretch from the lightcurve fits multiplied by $\alpha$.

- A term due to the uncertainty in redshift. This includes an assumed peculiar velocity dispersion of $300 \mathrm{~km} \mathrm{~s}^{-1}$ and redshift measurement errors .

- $\sigma_{\text {int }}$ magnitudes of intrinsic variation determined by fits to the low-redshift Hubble diagram.

At high redshift the redshift measurement errors are taken to be 0.001 when the redshift was measured from host galaxy lines and 0.01 when measured from SN features, as in P99 and K03. The intrinsic variation is assumed to be distributed as a Gaussian, and is determined by performing Hubble fits with low redshift SNe and finding the value that results in a $\chi^{2}$ per degree of freedom of 1. A Monte-Carlo simulation was used to calculate the errors associated with this estimate by generating 100,000 realizations of a nearby SN sample with identical properties to the actual one (redshift distribution and photometry errors). For $B_{B V 0.6}$ with stretch correction, $\sigma_{\text {int }}=0.12_{-0.04}^{+0.03}$ mag. Two additional estimators for $\sigma_{i n t}$ were considered: the RMS corrected for photometry errors and peculiar velocities, and the maximum-likelihood (ML) estimator for this problem. All three agree, although the ML and $\chi^{2}$ estimators are considerably more efficient than the corrected RMS. We note that this value for $\sigma_{i n t}$ is slightly higher than that given in W03; the values there were based on samples with tighter color cuts.

\footnotetext{
${ }^{1}$ The definition of $\mathcal{M}$ used here differs slightly from that of P99 and K03 in that all of the constants have been absorbed, including c.

${ }^{2}$ This could be verified prior to unblinding for $\mathcal{M}$ and $\alpha$, but the confirmation of this statement for $\Omega_{m}$ and $\Omega_{\Lambda}$ was only available after unblinding. If the final cosmology had disagreed very strongly with previous results, this would have led to problems with the blindness procedure. Fortunately, this turned out not to be the case.
} 


\subsection{Cuts on the Supernova Sample}

The procedure used to estimate the systematic errors in this paper is an extension of that used by P99 and K03 and differs only in that we have endeavored to be even more methodical in our exploration of changes to the fits. For this paper we specify a primary fit defined by a set of cuts, which are designed to be fairly loose while still removing SNe with obviously bad data or that provide no useful constraint on the cosmological parameters. We then explore the effects of changing these cuts in great detail and use the information thus gleaned to estimate the systematic errors. As we discuss below, altering most of these cuts has little effect on the final result, but this systematic exploration raises the specter of an unconscious fine-tuning to obtain the expected result. To circumvent this possibility we have performed a blind analysis, as detailed in 6.3 .

The cuts can roughly be split into two categories: data quality and analysis cuts. Not all are used in every fit considered. Their values for the primary fit are summarized in table 4. More complete descriptions are provided below. The same cuts are applied when determining the sample of SNe that are used to measure the intrinsic distribution of $\beta_{B V}$.

There are four data quality cuts:

- A cut on the minimum number of points in the linear cmagic region. As long as the date of maximum is well known, it is not necessary to have more than one point. ${ }^{3}$

- A cut on the maximum allowable error on $B_{B V 0.6}$. Objects with very poorly determined magnitudes add little statistical weight to the cosmology fit but make the Hubble diagram more difficult to read and in general obfuscate the result.

- A cut on the maximum allowable error in the date of maximum. This is used because the date of maximum is used to specify the points that are in the linear CMAGIC region. Points that fail this cut usually fail the next cut as well.

- A cut on the maximum allowable gap (in rest frame days) between the nearest point in either $B$ or $V$ and the date of $B$ maximum. If this gap is too large, the date of maximum, stretch, and maximum magnitude can easily be incorrect. This arises because the error in the light-curve template itself is currently not fully taken into account.

There are four analysis cuts:

- A minimum redshift cut for the cosmology fit. It is ignored when the sample of SNe used to determine the intrinsic $\beta_{B V}$ distribution is determined.

- A maximum redshift cutoff for the cosmology fit, which is not used in the primary fit.

\footnotetext{
${ }^{3}$ Technically a floor of 2 points is used when the slope distribution sample is determined, but this has no effect because all of the low redshift SNe have 2 or more points in the linear region.
} 
- A maximum allowable color excess at $B$ maximum when compared with the color model of K03. This can be interpreted as an extinction cut.

- A minimum allowable stretch value. SNe with best fit values below this are removed from the sample for the reason discussed below.

We find that our estimates for the cosmological parameters from $B_{B V 0.6}$ are relatively insensitive to changes in the cut on the color excess, but the same cannot be said of the $m_{B}$ fits. Because we seek to compare the CMAGIC results directly with the $m_{B}$ results, it is useful to choose a value of the color cut that can be used for both fits. Therefore, we have chosen to use the same cut as Knop et al. (2003) $(<0.25)$ in the primary fit.

A minimum stretch cut of 0.7 is applied to our primary fit sample because our $K$-corrections may not be reliable for extremely low stretch SNe, as their spectra display strong Ti features that are not well represented by our spectral template (Nugent et al. 2002). We require spectroscopic identification for our sample. There is only one SN that passes the other cuts but does not have a firm spectroscopic ID: SN 2001fo from Barris et al. (2004). As was the case in K03 and P99, SN $1997 \mathrm{O}$ has been manually excluded from our sample. When included it is a $7 \sigma$ outlier from the best fit cosmology. Two of the low-redshift SN in our sample (SN 1997br and SN 1997bp) appear to have internal inconsistencies in their photometry, displaying a far higher degree of scatter both in light-curve and CMAGIC fits than can be explained by their quoted photometric errors. ${ }^{4}$ We have taken the conservative approach of removing them from the sample. When included, they have no impact on the cosmological parameters. In addition to these cuts, the maximum redshift of SNe that are used to measure the $\beta_{B V}$ distribution is specified by another cut.

There are $119 \mathrm{SNe}$ at redshifts greater than 0.01 of the $131 \mathrm{SNe}$ in our baseline sample. Lower redshift SNe can also be included in our fits, but add essentially no statistical weight because of the dominance of their peculiar velocity errors. They are still useful for measuring the intrinsic slope distribution. The data quality cuts at the levels of the primary fit eliminate 62 of the SNe from the primary sample, and the analysis cuts remove five more. 53 are at $z>0.1$, of which 28 are eliminated by the quality cuts and four by the analysis cuts. We have explored the effects of both relaxing and tightening the cuts in a systematic fashion. Many of the SNe fail multiple cuts, and the cuts are not applied in any order, so it would be misleading to specify the number of SNe removed by each cut. However, a list of which SNe are removed by each cut is potentially interesting, and is provided in appendix C]

\footnotetext{
${ }^{4}$ The $\chi^{2}$ per degree of freedom for the CMAGIC fits to SN1997bp and SN1997br are around 4, which is particularly striking because for the majority of SNe Ia the $\chi^{2}$ per degree of freedom is considerably less than one.
} 


\subsection{Blindness}

"Experimenter bias" occurs when an analysis is affected by the expectations of the experimentalist. Such bias is frequently unconscious, and can take quite subtle forms. For example, a result that disagrees strongly with a previous result is frequently subject to more scrutiny than one that appears to be in agreement. This may bias an experimenter into being more likely to find errors that cause their result to disagree with expectations while making it less likely that they will discover errors that have the opposite effect. Since the research process has a natural termination point (publication), if the decision to stop analyzing a result is at all influenced by the value of the result, a bias will be introduced. A nice summary of these issues can be found in Heinrich (2003). It has long been recognized that a useful technique for mitigating experimenter bias is to hide the final results of the experiment from the experimenter for as long as possible. This is known as blind analysis. Such an approach is particularly useful in an analysis with a substantial number of cuts, such as that presented here. In the medical fields double blind procedures (which hide some details of the experiment from both the test subject and the experimenters) are used almost as a matter of course. Naturally, hiding the details of the experiment from the subject is not of great concern in astronomical research.

A critical point is that these techniques do not seek to completely hide all information during the analysis. In fact, the goal is to hide as little information as possible while still acting against experimenter bias. Human judgment and scientific experience continue to play a critical role in a blind analysis. One does not mechanically carry out the steps of the analysis and then publish the results. All that a blind analysis does is prevent unconscious misuse of particular types of information during the analysis process. The kind of data that are excluded from consideration (namely, the final answer derived from each option under consideration) is invariably that which no reasonable scientist would allow to consciously influence his or her decision making process. However, subconscious effects are still present, and this is what this approach helps prevent.

Specifically, it is important to design the blindness technique such that subsidiary diagnostics are available even while hiding the final answer. Errors are initially present in any analysis, and it is important that even while the result remains blinded mechanisms are available to catch these problems. Specifically, our goal is to hide the values of $\Omega_{m}$ and $\Omega_{\Lambda}$ until the cuts and fitting procedures have been finalized, while preserving as much ancillary information as possible. In particular, our method preserves the residuals of individual SNe with respect to the Hubble line, which is extremely useful while diagnosing the fits. For example, an error in the $K$-corrections might result in all SNe in a given redshift range departing significantly from the Hubble line. This problem would still be detectable in our blinded fits. In addition, the method preserves the shifts in $\Omega_{m}, \Omega_{\Lambda}$ between fits to different subsamples - if excluding a particular SN causes the unblinded result to shift by $\Delta \Omega_{m}=0.1, \Delta \Omega_{\Lambda}=0.2$, the blinded result shifts by the same amount, which is important when investigating systematic errors.

The technique used here is based on altering the true fit estimates. Hidden, but fixed, offsets 
are added to $\Omega_{m}$ and $\Omega_{\Lambda}$, and this change is propagated through to the $B_{B V 0.6}$ values. In essence the cosmological parameters are fitted twice, with the magnitudes modified between fits, but the results of the first fit are never output. Because it would be possible to circumvent the blindness if the real $B_{B V 0.6}$ values were known, these values must be kept hidden. All of the programs used to plot CMAGIC diagrams add random offsets to the $B$ magnitudes for display purposes. Furthermore, the CMAGIC fitter and cosmology fitter are integrated so that the true $B_{B V 0.6}$ values are not output.

The expression for the luminosity distance cannot be evaluated in terms of simple functions except in limited cases, so the magnitude modification is calculated numerically. The results of the first, unmodified, fit are marginalized to determine the secret true measured values $\Omega_{m T}$ and $\Omega_{\Lambda T}$. The hidden offsets are then applied to these values, and the difference in magnitudes between the two cosmologies is calculated and applied. If $\Delta \Omega_{m}$ and $\Delta \Omega_{\Lambda}$ are the hidden offsets, then the following function is added to $B_{B V 0.6}$ for each $\mathrm{SN}$ :

$$
\Delta B_{B V 0.6}(z)=5 \log _{10} \mathcal{D}_{L}\left(z, \Omega_{m T}+\Delta \Omega_{m}, \Omega_{\Lambda T}+\Delta \Omega_{\Lambda}\right)-5 \log _{10} \mathcal{D}_{L}\left(z, \Omega_{m T}, \Omega_{\Lambda T}\right),
$$

where $\mathcal{D}_{L}$ is as in equation [3. The cosmological fit is then redone with the new magnitudes and this result is output. It is safe to output the modified magnitudes, which can be used to construct a Hubble diagram and to perform various tests on the fit.

The simplest method to choose the hidden offsets is to generate them randomly. This performs poorly in this case because there are several non-physical regions in the $\Omega_{m}, \Omega_{\Lambda}$ parameter space. Negative values of $\Omega_{m}$ result in a non-convergent luminosity distance integral. For high values of $\Omega_{\Lambda}$ the universe did not experience a Big Bang, but is instead rebounding from a previous bout of contraction (Carroll et al. 1992). In such a universe there is a maximum observable redshift, and if any of the SNe are at higher redshifts the luminosity distance expression cannot be evaluated. A randomly generated offset could easily push the cosmological parameters into one of these regions. Instead we have chosen to generate the hidden offsets by specifying the desired values of $\Omega_{m}$ and $\Omega_{\Lambda}$ for a particular SN sample (the primary fit). A special version of the cosmological fitter determines the offsets between a fit to the primary sample and the chosen value ${ }^{5} \Omega_{m}=1, \Omega_{\Lambda}=1.1$. These offsets are then used for all other fits.

As long as the resulting fit values for $\Omega_{m}$ and $\Omega_{\Lambda}$ are roughly equal to $\left(\Omega_{m T}+\Delta \Omega_{m}, \Omega_{\Lambda T}+\Delta \Omega_{\Lambda}\right)$ this preserves the residuals with respect to the fit by construction. Because the same hidden offsets are used for all fits, this approximately preserves relative shifts between different fits. The caveat is that, for a particular value of $\Omega_{m}$ and $\Omega_{\Lambda}$, the shape of the luminosity distance equation effectively weights SNe depending on their redshift, and therefore altering the values of these parameters may cause the relative shifts in the blinded fits to be slightly different than for the true values.

\footnotetext{
${ }^{5}$ These values were chosen to be sufficiently different from the results of previous analyses to force internal reviewers to psychologically confront the blindness scheme while remaining close enough to the expected values that the resulting error contours were not overly distorted.
} 
Therefore, the offsets are determined iteratively. However, as long as the hidden offset is relatively small, this effect is negligible. Tests on both previous data sets (specifically, the low-extinction primary subset of K03) and artificially generated data show that this procedure works in that the resulting cosmological parameter estimates are equal to the unblinded result plus the specified offset. The offset between the blind target values and the actual estimates for this analysis was somewhat larger than anticipated, so the specified offset does not quite match the actual shift. However, the relative shifts are preserved accurately over small distances, which allowed us to compare different fits to the same data prior to unblinding.

A similar procedure is followed in the $w$ fits, although a different set of offsets are used. Because problems related to non-physical regions of the parameter space are not as severe in this case, the offsets to $\Omega_{m}$ and $w$ were randomly generated from the ranges $[-0.2,0.2]$ and $[-0.4,0.4]$.

Should a mistake in the analysis be found after the result is unblinded, it should still be corrected. In this situation, one should publish both the corrected and uncorrected results and note the effects of the discovered error on the result. An example of this can be found in Akerib et al. (2004). We also note that it is important to determine the systematic errors prior to unblinding, or it would be possible to explain away any unexpected results by inflating them. This technique certainly does not prevent all types of bias, but it does provide an opportunity to improve the situation, and thus is worth pursuing.

\subsection{Complete Fitting Procedure (Blind)}

Our cosmological fits proceed in the following order:

- The SNe used to measure the intrinsic $\beta_{B V}$ distribution are determined by applying the specified cuts. The distribution of $\beta_{B V}$ is then calculated from these SNe.

- A one-parameter $\left(B_{B V 0.6}\right)$ CMAGIC fit is performed for all SNe in the data sample using a Monte-Carlo fitting technique that takes into account the distribution of $\beta_{B V}$ from the distribution calculated in the previous step. The fitted $B_{B V 0.6}$ values are not output.

- The cuts are applied again to determine the SNe used to measure $\Omega_{m}$ and $\Omega_{\Lambda}$. The same cuts are used, except for the redshift ranges in 6.2

- A cosmological fit is performed. Estimates for $\Omega_{m}$ and $\Omega_{\Lambda}$ are calculated but not output.

- The hidden offsets are read in and added to $\Omega_{m}$ and $\Omega_{\Lambda}$. A magnitude offset is applied to each SN based on equation 4

- The cosmology is refitted with the new magnitudes. These results are output.

- The altered magnitudes are used to construct a Hubble diagram. 
Once the blindness was removed, the fits were redone without the secret offset step. We have also performed fits using the maximum $B$ magnitude, $m_{B}$. Since these fits are not a principal result of this paper they can be performed in an unblinded fashion, allowing us to test our procedures.

\section{COSMOLOGICAL RESULTS}

Figure 7 shows the $\Omega_{m}, \Omega_{\Lambda}$ confidence regions of our primary fit, based on 31 nearby and 21 distant SNe Ia. An additional nine very nearby $\mathrm{SNe}(z<0.01)$ are used while determining the $\beta_{B V}$ distribution (for a total of 40). The resulting estimates for the cosmological parameters are $\Omega_{m}=1.26_{-0.51}^{+0.38}$ and $\Omega_{\Lambda}=2.20_{-0.67}^{+0.41}$. If we require a flat universe, consistent with recent CMB results, then $\Omega_{m}=0.19_{-0.06}^{+0.06}$. These confidence regions are comparable to those from P99 (but not as good as those from K03), despite the fact that fewer SNe are involved, due to the smaller value of $\sigma_{i n t}$ for CMAGIC. The fit residuals are shown in figure 8

$\Omega_{m}$ and $\Omega_{\Lambda}$ are not the natural variables for this measurement, as they are not independent for this data set. The result of our analysis is better expressed in the principal axes frame of the error ellipse $\Omega_{1} \equiv 0.790 \Omega_{m}-0.613 \Omega_{\Lambda}$ (the short axis) and $\Omega_{2} \equiv 0.613 \Omega_{m}+0.790 \Omega_{\Lambda}$ (the long axis). Roughly, $\Omega_{1}$ can be thought of as measuring acceleration and $\Omega_{2}$ as measuring geometry. Analyzing the results in this frame has considerable benefits while calculating systematic errors and when comparing the CMAGIC results to those derived from maximum magnitudes. In this frame the results of the primary fit are $\Omega_{1}=-0.349_{-0.131}^{+0.117}$ and $\Omega_{2}=2.502_{-0.838}^{+0.530}$. The values of the nuisance parameters are $\alpha=0.516_{-0.206}^{+0.193}$ and $\mathcal{M}=25.166_{-0.045}^{+0.049}$, and they are almost completely statistically independent. Magnitudes and redshifts are provided in table 1 for the low-redshift sample, and in table 2 for the high redshift sample. The $\chi^{2}$ of this fit is 49.5 for 52 degrees of freedom. In the next section we discuss variations of the cuts, which produce different sets of SNe. The stretch-luminosity relation is shown in figure 9. When compared with the $m_{B}$ relation (Fig. 13 of Knop et al. (2003), for example), the evidence for the utility of a stretch correction is much weaker for $B_{B V 0.6}$.

Our estimates for $w$ in a flat universe are shown in figure 10. These are combined with the measurement of the angular size of the baryon acoustic peak (BAP) in SDSS galaxy clustering statistics at $z=0.35$ (Eisenstein et al. 2005), which are quite complementary to the SN measurements. The resulting constraint is $w=-1.21_{-0.12}^{+0.15}$ and $\Omega_{m}=0.25_{-0.02}^{+0.02}$ (statistical errors only).

This is the first analysis that treats the combined data from the different SN groups in a fully consistent manner. Unlike Leibundgut (2001) or Riess et al. (2004), we find no significant evidence for anomalously blue colors in the high-redshift SN, even though this sample contains many of the same objects as those studies. Figure 4 shows the $B-V$ color at $B$ maximum for the low- and high-redshift primary fit sample. The highly negative color point from the high-redshift sample is due to (by far) the most poorly measured SN, SN 1997af, which has $E(B-V)_{B \max }=-0.24 \pm 0.24$. Excluding this point, the mean color of the low redshift sample is $(B-V)_{B \max }=0.045 \pm 0.027$ 
and that of the high redshift sample is $(B-V)_{B \max }=0.027 \pm 0.019$, where the standard errors are quoted.

\section{SYSTEMATICS}

We explore various systematic errors by performing alternate fits and comparing the results with our primary fit. Because of the way in which our blindness scheme is constructed, this comparison was possible before the final answer was known. As was the case in Knop et al. (2003), we find that the effects of most of the systematics act along the long axis of our error ellipse. They therefore do not significantly affect the value of the SN measurements for determining if the Universe is accelerating, but do substantially limit our ability to measure geometry. Fortunately, this is the dimension in which CMB measurements are extremely powerful.

There are two types of systematic error possible in this analysis. First, there are the systematics arising from alterations in the fitting procedures, $K$-corrections, etc. Second, there are those arising from the cuts applied to the sample. Ideally this second set would be handled by a complete Monte-Carlo simulation of the SN sample. Unfortunately, there are far too many pieces of information missing to make the results of such a study at all useful. In order to construct a believable Monte-Carlo, it would be necessary to have a reasonable understanding of the intrinsic luminosity and extinction distributions, which have not been convincingly measured. To make matters substantially worse, it would also be necessary to have a good understanding of the search and follow up strategy used to construct the SN sample. Because the sample used in this paper is primarily constituted of literature SN, a clear definition of the search techniques and procedures is simply not available. Providing the results of such a procedure would provide a misleading sense of accuracy. We therefore proceed by calculating the effects of changing the cuts applied to our sample over what we consider to be a reasonable range and combining the resulting shifts as an estimate of the systematic error. Clearly this procedure is somewhat subjective, but any credible improvement requires the availability of large, well defined SN samples such as those that should be provided by the SNfactory, SNLS, SDSS Supernova Survey, and ESSENCE.

The effects of these shifts can most precisely be stated in terms of the principal axes of the primary fit error ellipse, $\Omega_{1}$ and $\Omega_{2}$, which is the primary justification for their use. Recall that for the primary fit $\Omega_{1}=-0.349_{-0.131}^{+0.117}$ and $\Omega_{2}=2.502_{-0.838}^{+0.530}$ (statistical errors only). We follow the standard practice of adding the negative and positive shifts in quadrature when handling asymmetric errors (however, see Barlow (2003) for criticism of this procedure). The resulting systematic errors are ${ }_{-0.062}^{+0.060}$ on $\Omega_{1}$ (the short axis), ${ }_{-0.545}^{+0.476}$ on $\Omega_{2}$ (the long axis), and ${ }_{-0.049}^{+0.029}$ on the value of $\Omega_{m}$ in a flat universe. The shifts are summarized in table 5 , and detailed individually in the following sections. Some representative examples can be seen in figure 11. An essentially identical procedure has been carried out for the fit to $w, \Omega_{m}$ in a flat Universe, including the BAP constraint, resulting in systematics error estimates of ${ }_{-0.12}^{+0.07}$ on $w$ and ${ }_{-0.01}^{+0.01}$ on $\Omega_{m}$. Note that this only includes the systematics from the SN measurement. Unlike the $\Omega_{m}, \Omega_{\Lambda}$ fits, here the statistical errors are 
dominant, reflecting the more challenging nature of the $w$ measurement.

\subsection{Variation of Fitting Procedures}

There are many reasonable ways to alter the CMAGIC fitting procedures that result in slightly different values of the cosmological parameters. We have attempted to explore some of these variations.

P99 found that using a floating value of $\alpha$ when propagating the stretch error into the fit magnitude artificially inflates $\alpha$, as this decreases the $\chi^{2}$ by increasing the magnitude errors. Therefore, $\alpha$ was fixed for the purposes of error propagation. As in K03, we find no evidence for this effect. Fixing $\alpha$ at the estimate from the primary fit $(\alpha=0.5)$ has essentially no effect on the $\Omega_{m}, \Omega_{\Lambda}$ values except to shrink the error bars slightly, as expected. Not performing a stretch correction $(\alpha=0)$ shifts the error ellipse primarily along $\Omega_{2}$ by 0.06 . This is not included in the final value for the systematic error.

It is possible to include estimates about the error in the stretch and date of maximum in the CMAGIC fitting procedure, since they influence which points are included in the CMAGIC fit. A modified version of the fitting code has been used to investigate this possibility. This approach is considerably more expensive computationally, and for this data sample it turns out to make no difference. In our fits we have effectively assumed that $B$ and $B-V$ are independent variables. An alternative formulation of the linear relations that treats $B$ and $V$ as independent variables is possible. This also has no effect on the fit values (less than 0.005 mag for any SN).

The light-curve fitting procedure used in P99 differs slightly from that used here (and by K03) in that the fits to the $V$ band were performed fixing the stretch and date of maximum to the values derived from a $B$ only fit. This procedure arose from concerns that the rest frame $V$ light curves for the high-redshift sample are more poorly sampled than the rest frame $B$ light curves, which is not the case for the low-redshift sample. Thus, a light-curve fitting procedure that treats both bands on an equal footing might effectively introduce a bias in the fits. This is of considerably less concern for this data sample, since by its nature CMAGIC demands good $V$-band coverage, but to guard against this problem we re-calculated all of the lightcurve fits following this prescription, which affects the CMAGIC fits because it changes the values of the stretch and date of maximum. The resulting effect on the error contours was minor, and primarily towards larger values of $\Omega_{2}$ by 0.144 .

Variations in the $K$-corrections are investigated by considering alternative versions of the spectral template. In particular, we follow K03 by making use of a $U$-enhanced version of the template with $U-B=-0.5$ instead of -0.4 as in our primary fit. This shifts the error ellipse

primarily along the short axis, with $\Delta \Omega_{1}=-0.052$ (towards smaller values of $\Omega_{m}$ ). The $\chi^{2}$ worsens slightly to 50.9. This is, by far, the most significant source of uncertainty related to alterations in the fitting procedures. Simply treating this error as a statistical contribution to each $\mathrm{SN}$ is 
a completely inadequate representation of its effect on the cosmological results. Clearly, future projects would benefit substantially from additional constraints on the $U$-band behavior of SNe Ia.

\subsection{Variation of Cuts}

We considered both increasing and decreasing the cut values for all of the cuts described in 6.2. Here we only present those that had a measurable effect on the error ellipse or are interesting for some other reason.

Requiring SNe to have observations within 5 rest-frame days of maximum eliminates two low redshift SNe (SN 1998ab and SN 2000fa) and one at high redshift (SN 1996E), and induces a shift along the long axis by $\Delta \Omega_{2}=+0.139$. Loosening the requirement to 10 days adds one high-redshift SN (SN 2001jp), and results in a shift along the $\Omega_{1}$ axis of +0.024 towards higher values of $\Omega_{m}$. Changing the minimum allowable redshift to 0.015 from 0.01 has an extremely small effect on the fit results while eliminating six low redshift SNe. Halving (to 0.25) or tripling (to 1.5) the cut on the maximum allowable magnitude error alternately removes five high redshift $\mathrm{SNe}$ or adds one, but does not affect the results substantially, as one would expect given the low weight given SNe with such large errors.

Placing a substantially tighter cut on the color at maximum $\left[E(B-V)_{B \max } \leq 0.1\right.$, similar to that used for the low-extinction subset of K03] shifts the error contours by a substantial amount along the long axis (towards a flat universe) by $\Delta \Omega_{2}=-0.467$, eliminating three high and eight low redshift SNe. Using a color cut of 0.125 (half of the primary fit value) is not substantially different than using 0.1. Relaxing the color cut to 0.5 adds two high-redshift (SN 1998aw and SN 2002ad) and four low redshift $\mathrm{SNe}$, and moves the contours principally along the short axis by $\Delta \Omega_{1}=-0.048$. While less affected by extinction than $m_{B}$, CMAGIC is not completely unaffected. The analysis presented in this paper suggests that assumptions about the extinction law are not a significant systematic bias, and therefore future studies, including those that use CMAGIC, may benefit by applying an extinction correction. This must be weighed against the decrease in independence of the two magnitudes after correction.

Requiring that the date of maximum be known to better than 0.5 days removes a large number of high redshift SNe from the sample (nine), but has little effect except to inflate the error contours along the long axis. Relaxing the requirement to 2 days adds eight poorly measured high-redshift SNe and shifts the ellipse outwards along the long axis by $\Delta \Omega_{2}=+0.115$.

Requiring that there be at least two observations in the CMAGIC linear region, and hence providing some level of confidence that the CMAGIC relations are being obeyed, does have a non-negligible effect on the cosmological parameters. Three high-redshift SNe are eliminated (SN 1998as, SN 2002ab, and SN 2002kd), and the error ellipse shifts primarily outward along the long axis by $\Delta \Omega_{2}=+0.23$. Even when two points are required in the linear region, the quality of the high redshift data is such that the CMAGIC slope $\beta_{B V}$ cannot be usefully fitted to each SN. 
As can be seen from the above discussion, the primary systematic effect related to the cuts on the SN sample is associated with the extinction cut. A better understanding of the extinction distribution would help reduce this systematic considerably. Note that we do not apply an extinction correction, so we are more sensitive to the extinction cut than some other analyses - although they trade this off with sensitivity to extinction and the intrinsic peak color of SNe Ia. Fortunately, the systematics arising from the cut selection are primarily along the long axis of the error ellipse, and hence have little effect on our detection of acceleration.

\subsection{Other Systematics}

We have also considered limiting our low-redshift SN sample to only those from large, systematic SN studies in order to limit any systematic errors arising from differences in calibration. There are three major low-redshift samples: Hamuv et al. (1996), Riess et al. (1999a) and Jha et al. (2005). Excluding all nearby SNe that are not from one of the above three sources has a very minor effect.

To test the sensitivity of our results to individual SNe, we have performed a jack-knife test by removing each of the 21 high-redshift SNe individually and recalculating the cosmological fit. Our values for $\Omega_{m}$ and $\Omega_{\Lambda}$ are sensitive to the removal of SN 2001ix and SN 2002kd, both at the very high redshift end of the sample. Removing either of these SNe shifts the contours primarily along the long axis, although in opposite senses. Removing SN 2001ix results in a shift inward of $\Delta \Omega_{2}=-0.28$, and removing SN 2002kd shifts the contour outward by $\Delta \Omega_{2}=0.31$. Interestingly, their effects on the cosmological parameters nearly cancel. This analysis would benefit from additional SNe in this redshift range, but overall the results are reasonably robust.

Properly speaking, $\sigma_{\text {int }}$ should be another quantity that is marginalized over while performing the cosmological fits. To determine if this is necessary, we performed fits in which $\sigma_{\text {int }}$ was varied by $1 \sigma$ in each direction, and found that the effects on the cosmological parameters were negligible (less than $0.1 \sigma$ in $\Omega_{1}$ and $\Omega_{2}$ ).

Since all of the high-redshift supernovae (and many of those at low redshift) come from fluxlimited samples, they suffer from Malmquist bias (Malmquist 1936). We note that only a difference in the amount of Malmquist bias between the low- and high-redshift SN samples can affect the cosmological results. This effect is discussed extensively in P99 and K03, and we adopt the estimates contained therein for these samples: $0.01 \mathrm{mag}$ for P99 and $0.03 \mathrm{mag}$ for K03. P99 also estimated the Malmquist bias for the Hamuv et al. (1996) sample as 0.04 mag. The Riess et al. (1999a) and Jha et al. (2005) samples were primarily discovered using a galaxy catalog search, so they may suffer from little or no Malmquist bias ( $\mathrm{Li}$ et al. 2001). We therefore adopt a Malmquist bias of 0 mag for these samples. It is difficult to estimate the Malmquist bias for the remaining SNe in the low redshift sample, since they were discovered in a rather inhomogeneous fashion. However, since they constitute only a small fraction of the sample, the effects of any Malmquist bias on the 
cosmological parameters from this sample are expected to be negligible, and so we adopt a value of 0 mag. For the remaining portion of the high-redshift sample (approximately half) we provisionally use the same value as for the P99 SNe, 0.01 mag. To test the effects of this bias on our estimate, we apply the offsets to each sample and recalculate the fit. The resulting shift in the cosmological parameters is quite small, less than $0.1 \sigma$ in both dimensions.

Appendix $\mathrm{A}$ contains a discussion of the effects of the 'bump' in the CMAGIC diagram exhibited by some SNe. The effects of this systematic are negligible along both axes (less than 0.05 $\sigma)$.

\section{ANALYSIS OF RESULTS}

There are two channels available for analyzing the results of this paper. First, the estimates of the cosmological parameters can be considered in isolation. Second, the CMAGIC results can be compared with a maximum magnitude fit to the same SN. Several of the systematics should affect both samples equally (e.g., Malmquist bias); therefore, this comparison should be more precise. However, this requires that the covariance between $m_{B}$ and $B_{B V 0.6}$ be determined.

\subsection{Constraints on the Cosmological Parameters}

The results of a CMAGIC fit to currently published SN data strongly favor an accelerating Universe - in fact, more strongly than previous results based on $m_{B}$. Perhaps more interesting is that the fit contours depart mildly from a flat universe. In the principal axis frame, a flat universe corresponds to $\Omega_{2}=0.756 \pm 0.010$ for $\Omega_{m}=0.191$. Once systematics are taken into account, the disagreement is $1.75 \sigma$, which is expected to occur approximately $8 \%$ of the time due to random chance. A similar result was seen in the SN sample of Tonrv et al. (2003), although at a somewhat lower level of significance. Both results are interesting, but not yet strong enough to be of serious concern. One of the lessons of blind analyses is that $1.5+\sigma$ disagreements occur in science more frequently than our intuition, developed from exposure to non-blind experiments, often expects. ${ }^{6}$

The departure from flatness is driven by SNe at moderate redshifts $0.3<z<0.5$. The three with the highest pull are SNe 1998as, 1996k, and 1997ce. It is difficult to find any common thread between them. They come from three different papers, were observed with different telescopes (although SN 1998as and SN 1997ce were both partially observed with $H S T$ ), and their photometry was reduced by different authors using different techniques. Since they constitute the low-redshift end of their respective surveys, there may be a suspicion that they suffer from unusually high extinction. While SN 1998as does suffer from considerable host galaxy extinction $\left(A_{V}=0.49\right.$;

\footnotetext{
${ }^{6}$ See Heinrich (2003) $§ 4$ for further discussion.
} 
K03), the other two suffer from negligible extinction $\left(A_{V}=0.02\right.$ and 0.08 for SN 1996K and SN 1997ce, respectively; Riess et al. 2004). Note that removing each of these SNe individually has little effect on our results, as explained in 8.3

\subsection{Comparison of $B_{B V 0.6}$ and $m_{B}$ Results}

The results of an $m_{B}$ fit to the same $\mathrm{SN}$ as the primary are compared with the $B_{B V 0.6}$ fit in figure 12. The $\chi^{2}$ of this fit is 44.32 for 52 degrees of freedom, and the resulting estimates are $\Omega_{m}=1.08_{-0.69}^{+0.49}$ and $\Omega_{\Lambda}=1.65_{-0.91}^{+0.65}$, with a flat universe value of $\Omega_{m}=0.32_{-0.07}^{+0.07}$. The principal axes of this fit are almost identical to those of the CMAGIC fit, so it is useful to express them

in this frame. Here they correspond to $\Omega_{1}=-0.167_{-0.133}^{+0.146}$ and $\Omega_{2}=1.969_{-1.146}^{+0.787}$ (statistical errors only). Note that the $m_{B}$ fits agree somewhat better with a flat universe than the $B_{B V 0.6}$ fits.

If $m_{B}$ and $B_{B V 0.6}$ were equivalent (given current templates) we would expect $\alpha$ to be identical for the two methods. When comparing these numbers the marginalized, one-dimensional errors are appropriate instead of the outer extent of the $1 \sigma$ error contours quoted previously. For $B_{B V 0.6}$ $\alpha=0.516_{-0.206}^{+0.193}$, and for $m_{B}$ it is $\alpha=0.995_{-0.226}^{+0.253}$, a difference of $1.6 \sigma$. They are marginally inconsistent, but not at a significant level.

Directly comparing the $m_{B}$ and $B_{B V 0.6}$ cosmological results requires that the correlation between the two methods be measured, and then propagated into the cosmological parameter space. The details of this process are presented in Appendix $\mathbb{B}$. The result is that the correlation coefficients between the two fits are 0.34 along the $\Omega_{1}$ axis and 0.15 along $\Omega_{2}$.

While many of the systematic errors should affect $m_{B}$ and $B_{B V 0.6}$ equally, not all apply to both fits. For example, the number of points in the CMAGIC linear region is meaningless in an $m_{B}$ context. Furthermore, individual SNe may have quite different weights in the two fits, which partially removes the insensitivity to systematics. Both issues must be addressed before the results can be compared. The number of points in the linear region and the detectability of CMAGIC bumps at high redshift have already been discussed, and are summarized in table 5 . In addition, we expect that the effects of the $U-B$ color of the spectral templates will not be the same for both methods, since $m_{B}$ and $B_{B V 0.6}$ depend on color information in a very different fashion. Comparing the results of $m_{B}$ and $B_{B V 0.6}$ fits using the $U$-enhanced spectral templates as discussed in 8.1 we find that the residual difference due to this systematic is $\Delta \Omega_{1}=0.010, \Delta \Omega_{2}=0.151$. The effects of the differing weights can be addressed by performing a fit to $m_{B}$ where each $\mathrm{SN}$ is given the weight it has in the $B_{B V 0.6}$ fit, and vice-versa. It is not fair to include both values as systematics errors, since they are essentially measuring the same effect. Fortunately, they turn out to have almost identical effects. The short axis is brought into better agreement by a shift of $\Delta \Omega_{1}=0.054$ and the long axis by $\Delta \Omega_{2}=0.31$.

Putting these contributions together, and using the correlations given above, we find that the 
difference between the $m_{B}$ and $B_{B V 0.6}$ fits is

$$
\begin{aligned}
& \Delta \Omega_{1}=-0.182 \pm 0.097(\text { stat }) \pm 0.058(\text { sys }) \\
& \Delta \Omega_{2}=0.530 \pm 0.661(\text { stat }) \pm 0.414(\text { sys }) .
\end{aligned}
$$

The difference along the $\Omega_{1}$ axis amounts to $1.6 \sigma$, and along the $\Omega_{2}$ axis to $0.7 \sigma$. The major disagreement is along the short axis, as is obvious from figure 12, and a disagreement of this size or larger is expected to occur in $11 \%$ of measurements. Since $\Omega_{1}$ is essentially sensitive to acceleration, this amounts to the statement that the $B_{B V 0.6}$ results favor more acceleration at the $1.6 \sigma$ level. The differences along both axes can be combined into one measure by projecting them along the difference vector, defined by $\Omega_{3} \equiv-0.325 \Omega_{1}+0.946 \Omega_{2}$. Then the difference between the two fits is $\Delta \Omega_{3}=0.560 \pm 0.657$ (stat) \pm 0.410 (syst), a difference of $0.7 \sigma$.

A similar comparison is possible with the $\Omega_{m}, w$ fits. The result is shown in figure 13. The same sort of detailed comparison is not carried out here for several reasons. First, the difference is certainly not independent from the difference observed in $\Omega_{m}, \Omega_{\Lambda}$ space, so little additional information would be gained from this procedure. Second, because the current constraints on $\Omega_{m}$, $w$ from SN data alone are not well behaved (not closing off until very negative values of $w$ ), it is not useful to compare the two fits without the addition of additional constraints, here the BAP measurement, which is the same between the two fits.

\section{CONCLUSIONS}

CMAGIC provides some additional information that is not captured by the standard lightcurve template fitting techniques used to estimate $m_{B}$. This allows us to provide some additional constraints on the cosmological parameters. Furthermore, $B_{B V 0.6}$ should be affected differently by several potential evolutionary effects.

We have carried out the first blind analysis of the cosmological parameters using SN data, developing a technique to prevent experimenter bias by hiding the final result until the data cuts and analysis procedures are finalized. We find that the results of a CMAGIC fit broadly confirm our picture of an accelerating Universe. In fact, they favor a higher amount of acceleration than the $m_{B}$ results by approximately $1.6 \sigma$ (including systematics and the correlations between the two measurements). The $B_{B V 0.6}$ error contours differ from a flat Universe by $1.7 \sigma$ (including systematics), which would be interesting if it were more statistically significant.

The constraints on the cosmological parameters from a CMAGIC fit to 31 nearby and 21

distant SNe Ia are $\Omega_{m}=1.26_{-0.51}^{+0.38}, \Omega_{\Lambda}=2.20_{-0.67}^{+0.41}$ (statistical errors only). However, this is a poor frame for expressing the results. It is significantly more useful to instead quote the results as

$$
\begin{gathered}
\Omega_{1}=0.790 \Omega_{m}-0.613 \Omega_{\Lambda}=-0.349_{-0.131}^{+0.117}(\text { stat })_{-0.062}^{+0.060} \text { (syst) } \\
\Omega_{2}=0.613 \Omega_{m}+0.790 \Omega_{\Lambda}=2.502_{-0.838}^{+0.530}(\text { stat })_{-0.545}^{+0.476}(\text { syst })
\end{gathered}
$$


with

$$
\Omega_{m}=0.19_{-0.06}^{+0.06}(\text { stat })_{-0.05}^{+0.03} \text { (syst) }
$$

for a flat Universe, where the dark energy has been assumed to have a constant equation of state with $w=-1$, as is the case for a cosmological constant. The systematic errors have been estimated by considering a wide range of alternatives to the primary fit of this paper. The largest systematic error is the extinction cut, indicating that while CMAGIC has some benefits with respect to extinction by interstellar dust, we still have a great deal to learn about this issue. A direct comparison is also possible with an $m_{B}$ fit to the same $\mathrm{SN}$, which requires that the correlations between the two methods be estimated. After including the systematics and correlations, the difference between the two fits is almost exclusively along the short axis, with the CMAGIC fits favoring more acceleration by $1.6 \sigma$. Fitting for a constant value of $w$ in a flat Universe, the combination of the CMAGIC results with the angular scale of the BAP measured in Eisenstein et al. (2005) yields

$w=-1.21_{-0.12}^{+0.15}$ (stat) ${ }_{-0.12}^{+0.07}$ (supernova syst), $\Omega_{m}=0.25_{-0.02}^{+0.02}$ (stat) ${ }_{-0.01}^{+0.01}$ (supernova syst), consistent with a cosmological constant at the $1.2 \sigma$ level.

The currently available high redshift SN sample was not observed in an optimal fashion for CMAGIC. Out of the approximately 100 published high-redshift SNe light curves, only about 20 are useful for $B_{B V 0.6}$. As a result, the current data set does not place strong constraints on dust or evolutionary effects. This situation will change in this decade; within the next 5 years it should be possible to measure both $B_{B V 0.6}$ and $m_{B}$ for 1000 high-redshift SNe, at which point the comparison between $m_{B}$ and $B_{B V 0.6}$ will be extremely interesting.

The authors would like to thank Brian Schmidt for providing non- $K$-corrected light curves for SN 1997ce and SN 1997cj. This research has made use of the NASA/IPAC Extragalactic Database (NED) which is operated by the Jet Propulsion Laboratory, California Institute of Technology, under contract with the National Aeronautics and Space Administration.

\section{A. CMAGIC BUMPS}

An example of an SNe Ia with a bump feature is shown in the bottom of figure 1, Bumps seem to be associated with SNe with different $B$ and $V$ stretches (where the templates have been normalized such that the majority of $\mathrm{SNe}$ are well fitted with the same $B$ and $V$ stretch), in particular when $s_{V}<s_{B}$. In general, the probability of a bump increases with $B$ stretch. It is possible to find examples of SNe Ia with virtually the same stretch but where one has a bump and the other does not. This clearly indicates that SNe Ia do not constitute a one-parameter family, at least in terms of stretch, $\Delta m_{15}(B)$ or the MLCS parameter $\Delta$. Bumps are far more common in other filter combinations.

However, these matters do not concern us here. The important thing for the purposes of this paper is the effect of the bump on the cosmology fits. As noted previously, the presence of the bump 
has an effect on the starting and ending dates of the linear feature. With high-quality data it is trivial to detect the presence of a bump. Therefore, while this is not an issue with the low-redshift $\mathrm{SNe}$, it is a potential systematic in the cosmology fits due to the lower quality of the high-redshift data making bumps difficult to detect for some SNe. Fortunately, this turns out to have a relatively small effect for the present sample.

In order to quantify this effect, we attempted to determine the probability, as a function of stretch, that an SN has a bump by examining the low redshift sample. We find that all SNe with $s>1.1$ have a bump feature, and none with $s<0.8$ do. Between these extremes the probability of having a bump is an increasing function of stretch, but remains probabilistic. For $1.1<s<1.0$ approximately $50 \%$ of SNe Ia have bumps, and for $1.0<s<0.8$ only 1 out of 13 does. Applying this result to the high redshift sample, we see that there are six SNe in the first group and 14 in the second. One of the 14 (SN 1997ce) has a bump, consistent with the predicted fraction. As expected, individual filter fits to SN 1997ce show that the $V$ stretch is less than the $B$ stretch, with $s_{B}=0.932 \pm 0.025$ and $s_{V}=0.816 \pm 0.019$. The systematic effect, if any, will clearly arise from the first group, which consists of SNe 1995ba, 1997F, 1998aw, 1999fj, 2001ix, and 2002ad. The CMAGIC fits to SN 1998aw are not affected by the presence or absence of a bump, so it can be ignored for the purposes of this discussion.

In order to quantify the probability that each of these SNe has an undetected bump, we analyzed a handful of very well observed low redshift SNe that have a bump feature (SNe 1995D, 1995bd, 1998bu, and 1999ee) and used their CMAGIC diagrams to quantify the excess $B$ magnitude over the value predicted by the CMAGIC linear fit as a function of rest frame epoch. We then compared these values with the actual data points for the four high-redshift SNe in question, taking into account the observational errors and the dispersion of excess magnitudes in the bump. SN 1999fj, SN 2001ix, and SN 2002ad are inconsistent with a bump at greater than the $2.5 \sigma$ level. No strong statement can be made for SN 1995ba or SN 1997F. Therefore, these are the only two that need concern us.

This gives four possibilities, which occur with approximately equal probability. The case where neither has a bump is identical to our primary fit. In order to estimate the systematic error associated with the other possibilities, we performed and compared all four fits, obtaining results very similar to our primary fit. We find that the effects of this systematic on the current sample are $\Delta \Omega_{1}=0.005$ and $\Delta \Omega_{2}=-0.014$. This indicates that undetected bumps do not contribute substantially to the systematic error. The story is somewhat complicated, but we have been fortunate in that it does not affect the current result. Most future projects, which will obtain considerably more complete color coverage, should not have to worry about this issue. 


\section{B. CORRELATIONS BETWEEN THE $m_{B}$ AND $B_{B V 0.6}$ FITS}

In order to determine the correlation between the cosmological results of the $m_{B}$ and $B_{B V 0.6}$ fits, it is first necessary to determine the correlations between $m_{B}$ and $B_{B V 0.6}$ values for each SN. There are two components to this correlation: that induced by the fitting procedures, and that intrinsic to the physics of SNe Ia and their environment (extinction, etc.). The former can be determined individually for each $\mathrm{SN}$, and is seen to vary considerably depending on the distribution of observations, while we are forced to assume that the latter is constant across the SN sample.

Since current light-curve templates do not adequately reproduce the CMAGIC relations, the fit correlation must be determined by a Monte Carlo process. For every SN, 1000 realizations are generated using the actual photometric errors and observed epochs. For each realization $m_{B}$ and $B_{B V 0.6}$ are fitted independently, and the correlations are estimated from the resulting distributions. After stretch correction, the correlation between $m_{B}$ and $B_{B V 0.6}$ is small and positive, with mean correlation coefficients of $\langle\rho\rangle=0.150$ at low redshift and $\langle\rho\rangle=0.144$ for distant SNe. The distributions are shown in figure 14. Furthermore, the correlation between stretch and $B_{B V 0.6}$ is quite weak, justifying the assumption that they are uncorrelated in the CMAGIC fitting procedure $(\langle\rho\rangle=0.097)$.

In order to estimate the residual resulting from the intrinsic heterogeneity of SNe Ia, the best tool is to consider the residual versus residual plot, shown in figure [15. Note that these residuals are with respect to different fits with different values of the cosmological parameters. It is clear that they are correlated, although this is much less true of the high redshift sample: $\operatorname{cov}\left[m_{B}, B_{B V 0.6}\right]_{\text {lowz }}=0.020$ and $\operatorname{cov}\left[m_{B}, B_{B V 0.6}\right]_{\text {highz }}=0.0076$, where cov denotes the covariance between the two quantities. These values correspond roughly to $\rho=0.55$ and $\rho=0.34$, respectively. It is not surprising that the low-redshift sample shows considerably more correlation because of the dominant role of peculiar velocity errors, which affect $m_{B}$ and $B_{B V 0.6}$ identically.

To estimate the intrinsic correlation it is necessary to subtract the effects of both the peculiar velocity and the correlations induced by the light-curve and CMAGIC fitting procedures. If $r_{m_{B}}$ and $r_{B_{B V 0.6}}$ denote the residuals from the fit, then, using the low-redshift approximation for $\mathcal{D}_{L}$ (which is appropriate because peculiar velocities have a negligible effect at high redshift), and noting that the stretch and redshift are anti-correlated,

$$
\begin{aligned}
\operatorname{cov}\left[r_{m_{B}}, r_{B_{B V 0.6}}\right]= & \operatorname{cov}\left[m_{B}, B_{B V 0.6}\right]+\alpha_{m_{B}} \operatorname{cov}\left[s, B_{B V 0.6}\right]+ \\
& \alpha_{B_{B V 0.6}} \operatorname{cov}\left[s, m_{B}\right]+\sigma_{s}^{2} \alpha_{m_{B}} \alpha_{B_{B V 0.6}}+\left(\frac{5}{\log 10} \frac{\sigma_{z}}{z}\right)^{2}+ \\
& \frac{5}{\log 10}\left(\alpha_{m_{B}}+\alpha_{B_{B V 0.6}}\right) \frac{\sigma_{z}}{z} \sigma_{s}+\operatorname{cov}\left[\mathcal{M}_{m_{B}}, \mathcal{M}_{B_{B V 0.6}}\right]
\end{aligned}
$$

Here the correlations between stretch, $m_{B}$, and $B_{B V 0.6}$ are those arising from the fitting procedure

only. The desired quantity is $\operatorname{cov}\left[\mathcal{M}_{m_{B}}, \mathcal{M}_{B_{B V 0.6}}\right]$, the correlation between the absolute magnitudes modulo the Hubble constant. Note that the stretch-corrected covariance shown in figure 14 is not 
appropriate here because the contributions of stretch are handled separately. More than half of the measured covariance in the low redshift sample (0.013) comes from peculiar velocity errors, which have essentially no effect on the high redshift sample. We find that $\operatorname{cov}\left[\mathcal{M}_{m_{B}}, \mathcal{M}_{B_{B V 0.6}}\right]_{\text {lowz }}=$ 0.0072 and $\operatorname{cov}\left[\mathcal{M}_{m_{B}}, \mathcal{M}_{B_{B V 0.6}}\right]_{\text {highz }}=0.0044$, which correspond to $\rho=0.37 \pm 0.14$ and $\rho=$ $0.22 \pm 0.21$, respectively. These are consistent, and therefore the overall correlation coefficient for the intrinsic scatter is taken to be $\rho=0.32 \pm 0.12$.

Next it is necessary to propagate this covariance into the cosmological parameter space. This is far from straightforward. While it might be tempting to simply assume that the intrinsic correlation is the dominant one, and that this can therefore be taken as the correlation between the $\Omega_{1}$ values of the two fits, there is no way to justify this assumption. The correlation at low redshift is dominated by the peculiar velocity errors, and it is unclear how important this is in the context of the cosmological parameters. Furthermore, different SNe have different weights, both because of their observational errors and because $\mathrm{SNe}$ at different redshifts have different influences in the $\Omega_{m}, \Omega_{\Lambda}$ parameter space.

In order to determine the effects of these correlations on $\Omega_{1}, \Omega_{2}$, a Monte-Carlo simulation was carried out on the SN samples. The covariances between stretch, $m_{B}$ and $B_{B V 0.6}$ from the fitting procedures were calculated for each supernova as described above, to which were added the measured intrinsic correlation coefficient of 0.32 . This simulation also incorporated the effects of redshift errors including the assumed peculiar velocity of $300 \mathrm{~km} \mathrm{~s}^{-1}$.

Generating 2500 realizations required approximately 4 days on a fast workstation. The corresponding correlation coefficients for the $\Omega_{1}$ and $\Omega_{2}$ axes are $\rho_{11}=0.34 \pm 0.02$ and $\rho_{22}=0.15 \pm 0.02$. The correlation is not evenly distributed between the two axes, acting primarily along the short axes of the error ellipses. Since these correlations are positive, they act to increase the significance of the difference between the two fits. The same data set can be used to verify that $\Omega_{1}$ and $\Omega_{2}$ are uncorrelated, yielding $\rho_{12 m_{B}}=-0.07$ and $\rho_{12 B_{B V 0.6}}=0.07$.

\section{SUPERNOVAE REMOVED BY EACH CUT}

This section presents a list of the SNe removed by each cut at the values specified in the primary fit. Note that these cuts are not applied in any order, and therefore some SNe fail multiple cuts. Furthermore, some of the cuts are correlated. For example, an SN that does not have any data within 7 days of $B$ maximum is unlikely to have a well-determined date of maximum.

The following SNe do not have any points in their CMAGIC linear region: SNe 1995ar, 1995aw, 1995ay, 1995az, 1996cf, 2001iw, and 2002P. These were at redshifts too low to be used in the cosmology fit (although some were used to determine the intrinsic $\beta_{B V}$ distribution): SN1990N, SN1994ae, SN1995D, SN1995al, SN1996X, SN1996Z, SN1997bp, SN1997bq, SN1997br, SN1998bu, SN1998dh, SN1999ac, SN1999by, SN1999cl, SN1999gh, SN2000E, SN2001el, SN2002bo. The following SN did not have data with 7 rest frame days of B maximum: SN1990T, SN1990Y, SN1991S, 
SN1991U, SN1991ag, SN1992bg, SN1992bk, SN1993ae, SN1993ah, SN1994Q, SN1997bq, SN1998ec, SN1999gh, SN2000bh, SN2000ce, SN2001jn, SN2001jp, and SN2002P. These SNe did not have a well determined date of maximum: SN1992ae, SN1992au, SN1992bk, SN1993B, SN1993ah, SN1994G, SN1995ak, SN1995aq, SN1995ar, SN1995ax, SN1995ay, SN1996I, SN1996U, SN1996Z, SN1996cm, SN1997K, SN1997S, SN1999fn, SN2000bh, SN2001hx, SN2001hy, SN2001jb, SN2001jf, SN2001jn, and SN2002P. The following SNe had stretch values below the minimum cutoff, and were removed for the reasons discussed in 6.2 SN1992au, SN1998bp, SN1998de, SN1999by. SN1996U, SN1997K, SN1997am, SN1999ff, SN2001hx, SN2001hy, and SN2001jb have errors on $B_{B V 0.6}$ that exceeded 0.5 mag. SN1990Y, SN1992J, SN1993H, SN1995E, SN1995bd, SN1996C, SN1996Z, SN1996bo, SN1997br, SN1998aw, SN1998bu, SN1999cl, SN1999ee, SN1999fw, SN1999gd, SN2000ce, SN2001jn, SN2002ad, and SN2002bo have measured color excesses larger than the 0.25 mag cut value. As discussed in $\$ 6.2$, there were 3 additional SN removed by hand from the sample for various reasons: SN1997O, SN1997br, and SN1997bp.

\section{REFERENCES}

Aguirre, A. 1999, ApJ 525, 583.

Akerib, D. S. et al. 2004, Phys. Rev. Lett. 93, 1301

Aldering, G. et al. 2004, preprint (astro-ph/0405232)

Astier, P. et al. 2005, å in press

Barlow, R. 2003, preprint (physics/0306113s)

Barris, B.J. et al. 2004, ApJ 602, 571

Benetti et al. 2004, MNRAS 348, 261

Bessell, M.S. 1990, PASP 102, 1181

Cardelli, J.A., Clayton, G.C. and Mathis, J.S. 1989, ApJ 345, 245

Carroll, S.M., Press, W.H., and Turner, E.L. 1992, ARA\&A 30, 499

Domígues, I., Höflich, P., and Straniero, O. 2001, ApJ 557, 279

Draine, B.T. 2003, ARA\&A 41, 241

Eisenstein, D.J. et al. 2005, ApJ 633, 560

Fitzpatrick, E.L. 1999, PASP 111, 63

Garavini, G. et al. 2005, in preparation. 
Garnavich et al. 1998, ApJ 493, L53 (lightcurves available from astro-ph/9710123)

Goldhaber, G. et al. 2001, ApJ 558, 359

Guy, J. et al. 2005, å in press

Hamuy, M. et al. 1996, AJ 112, 2408

Hamuy, M. et al. 2000, AJ 120, 1479

Hatano, K., Branch, D., and Deaton, J. 1998, ApJ 502, 177

Heinrich, J.G. 2003, CDF Memo 6576

Höflich, P., Nomoto, K., Umeda, H., and Wheeler, J.C. 2000, ApJ 528, 590

Hook, I.M., Howell, D.A. et al. 2005, AJ, in press

James, F. and Roos, M. 1975, Compt. Phys. Comm. 10, 343

Jha, S. et al. 2005, AJ in press

Kim, A., Goobar, A., and Perlmutter, S. 1996, PASP 108, 190

Knop, R.A. et al. 2003, ApJ 598, 102

Krisciunas, K. et al. 2000,ApJ 539, 658

Krisciunas, K. et al. 2001, AJ 122, 1616

Krisciunas, K. et al. 2004, AJ 127, 1664

Leibundgut, B. 2001 ARA\&A 39, 67

Li, W. et al. 2001, ApJ 546, 734

Malmquist, K.G. 1936, Stockholm Observatory Medd., no. 26

Matheson, T. et al. 2005, AJ 129, 2352

Modjaz, M. et al. 2001, PASP 113, 308

Nobili, S. et al. 2003, å 404, 901

Nobili, S. et al. 2005, å 437, 789

Nugent, P., Kim, A., and Perlmutter, S. 2003, PASP 114, 803

Padmanabhan, N. et al. 2005, Phys. Rev. D 72, 043525

Perlmutter, S. et al. 1997, ApJ 483, 565 
Perlmutter, S. et al. 1998, Nature, 391, 51

Perlmutter, S. et al. 1999, ApJ 517, 565

Phillips, M.M. 1993, ApJ 413, L105

Phillips, M.M. et al. 1999, AJ 118, 1766

Pinto, P.A., Smith, C.R., and Garnavich, P.M. 2004, AAS meeting 205, \#108.20

Riess, A.G., Press, W.,H., and Kirshner, R.P. 1996, ApJ 473, 88

Riess, A.G. et al. 1998, AJ 116, 1009

Riess, A.G. et al. 1999, AJ 117, 707

Riess, A.G., Filippenko, A.V., Li W., and Schmidt B.P. AJ 116, 1009

Riess, A.G. et al. 2000, ApJ 536, 62.

Riess, A.G. et al. 2004, ApJ 607, 665

Sako, M. et al. 2005, preprint (astro-ph/0504455)

Schlegel, D.J., Finkbeiner, D.P., and Davis, M. 1998, ApJ 500, 525

Schmidt, B. et al. 1998, ApJ 507, 46

Simon, B. and Shaw, R.A. 1996, in ASP Conf. Ser. 101: Astronomical Data Analysis Software and Systems V, ed. G.J. Jacoby and J. Barnes, 183

Spergel, D.N. et al. 2003, ApJS 148, 175

Strolger, L.G. et al. 2002, AJ 124, 2905

Sullivan, M. et al. 2002, MNRAS 340, 1057

Suntzeff, N.B. et al. 1999, AJ 117, 1175

Tegrmark, M. et al. 2004, Phys. Rev. D 69, 103501

Tonry et al. 2003, ApJ 594, 1

Tripp, R. and Branch, D. 1999, ApJ 525, 209

Vinko, J. et al. 2003, A\&A 397, 115

Wang, L., Goldhaber, G., Aldering, G., and Perlmutter, S. 2003, ApJ 590, 944 
Table 1. Low redshift SNe used in primary fit

\begin{tabular}{|c|c|c|c|c|}
\hline IAU Name & $z_{\text {hel }}{ }^{\mathrm{a}}$ & stretch & $B_{B V 0.6^{\mathrm{b}}}$ & Reference \\
\hline SN1990O & 0.031 & $1.087(032)$ & $17.530(067)$ & 1 \\
\hline SN1990af & 0.050 & $0.750(010)$ & $18.894(078)$ & 1 \\
\hline SN1992ag & 0.026 & $0.959(022)$ & $17.222(056)$ & 1 \\
\hline SN1992al & 0.014 & $0.929(013)$ & $15.838(083)$ & 1 \\
\hline SN1992bc & 0.020 & $1.079(007)$ & $16.738(062)$ & 1 \\
\hline SN1992bh & 0.045 & $1.057(024)$ & $18.697(050)$ & 1 \\
\hline SN1992bl & 0.043 & $0.845(021)$ & $18.556(065)$ & 1 \\
\hline SN1992bo & 0.018 & $0.744(007)$ & $16.918(049)$ & 1 \\
\hline SN1992bp & 0.079 & $0.897(021)$ & $19.634(086)$ & 1 \\
\hline SN1992bs & 0.063 & $1.025(017)$ & $19.568(071)$ & 1 \\
\hline SN1993O & 0.052 & $0.927(020)$ & $18.912(034)$ & 1 \\
\hline SN1993ag & 0.049 & $0.940(027)$ & $18.839(064)$ & 1 \\
\hline SN1994M & 0.023 & $0.883(025)$ & $17.422(084)$ & 2 \\
\hline SN1994S & 0.015 & $1.052(024)$ & $16.181(102)$ & 2 \\
\hline SN1996bl & 0.036 & $1.014(014)$ & $17.879(029)$ & 2 \\
\hline SN1996bv & 0.017 & $1.039(020)$ & $16.225(030)$ & 2 \\
\hline SN1997E & 0.013 & $0.821(006)$ & $16.232(038)$ & 3 \\
\hline SN1998V & 0.018 & $0.962(040)$ & $16.389(068)$ & 3 \\
\hline SN1998ab & 0.027 & $0.958(006)$ & $17.212(036)$ & 3 \\
\hline SN1998es & 0.011 & $1.075(014)$ & $15.074(048)$ & 3 \\
\hline SN1999aa & 0.014 & $1.098(004)$ & $16.135(017)$ & 4 \\
\hline SN1999aw & 0.038 & $1.358(008)$ & $18.242(035)$ & 5 \\
\hline SN1999dk & 0.015 & $1.089(010)$ & $15.862(020)$ & 6 \\
\hline SN1999dq & 0.014 & $1.060(004)$ & $15.498(076)$ & 3 \\
\hline SN1999ek & 0.018 & $0.895(007)$ & $16.573(049)$ & 7 \\
\hline SN1999gp & 0.027 & $1.141(004)$ & $17.222(064)$ & 6 \\
\hline SN2000ca & 0.024 & $1.007(016)$ & $17.137(067)$ & 7 \\
\hline $\mathrm{SN} 2000 \mathrm{dk}$ & 0.017 & $0.720(004)$ & $16.394(037)$ & 3 \\
\hline SN2000fa & 0.021 & $0.972(007)$ & $17.025(062)$ & 3 \\
\hline SN2001V & 0.015 & $1.119(017)$ & $15.769(110)$ & 8 \\
\hline SN2001ba & 0.029 & $1.049(014)$ & $17.669(042)$ & 7 \\
\hline
\end{tabular}

${ }^{a}$ Heliocentric redshift.

${ }^{\mathrm{b}}$ Does not include $\sigma_{\text {int }}$

Note. - SNe in primary cosmology fit, not including SNe not in the Hubble flow used to measure the 
slope distribution.

References. - (1) Hamuv et al. (1996), (2) Riess et al. (1999a), (3) Jha et al. (2005), (4) Krisciunas et al. (2000), (5) Strolger et al. (2002), (6) Krisciunas et al. (2001), (7) Krisciunas et al. (2004), (8) Vinko et al. (2003) 
Table 2. High redshift SNe used in primary fit

\begin{tabular}{lcccc}
\hline \hline IAU Name & $z_{h e l}$ a & stretch & $B_{B V 0.6}{ }^{\mathrm{b}}$ & Reference \\
\hline SN1995K & 0.479 & $0.956(046)$ & $24.276(220)$ & 1 \\
SN1995ba & 0.388 & $0.999(052)$ & $24.025(267)$ & 2 \\
SN1996E & 0.430 & $0.940(005)$ & $23.572(156)$ & 3 \\
SN1996K & 0.380 & $0.888(013)$ & $24.169(166)$ & 3 \\
SN1997F & 0.580 & $1.034(070)$ & $24.861(349)$ & 2 \\
SN1997H & 0.526 & $0.883(051)$ & $24.242(478)$ & 2 \\
SN1997P & 0.472 & $0.898(039)$ & $24.610(487)$ & 2 \\
SN1997ai & 0.450 & $0.918(112)$ & $23.876(283)$ & 2 \\
SN1997af & 0.579 & $0.846(050)$ & $24.655(508)$ & 2 \\
SN1997ce & 0.440 & $0.932(025)$ & $24.327(062)$ & 4 \\
SN1997cj & 0.500 & $0.925(021)$ & $24.453(077)$ & 4 \\
SN1997eq & 0.540 & $0.947(026)$ & $24.514(194)$ & 5 \\
SN1998as & 0.355 & $0.961(023)$ & $23.786(100)$ & 5 \\
SN1998ax & 0.497 & $1.156(032)$ & $24.447(115)$ & 5 \\
SN1998ba & 0.430 & $0.975(022)$ & $24.241(091)$ & 5 \\
SN1999fj & 0.816 & $1.037(040)$ & $25.517(273)$ & 6 \\
SN2000fr & 0.543 & $1.100(020)$ & $24.542(079)$ & 5 \\
SN2001iv & 0.397 & $0.977(004)$ & $23.720(091)$ & 7 \\
SN2001ix & 0.711 & $1.025(052)$ & $24.937(159)$ & 7 \\
SN2002ab & 0.423 & $0.924(015)$ & $23.872(214)$ & 7 \\
SN2002kd & 0.735 & $0.907(013)$ & $25.385(114)$ & 8 \\
\hline
\end{tabular}

${ }^{a}$ Heliocentric redshift.

${ }^{\mathrm{b}}$ Does not include $\sigma_{\text {int }}$

Note. - SNe in primary cosmology fit, not including SNe not in the Hubble flow used to measure the slope distribution.

References. - (1) Schmidt et al. (1998), (2) Perlmutter et al. (1999), (3) Riess et al. (1998), (4) B. Schmidt, private communication, (5) Knop et al. (2003), (6) Tonrv et al. (2003), (7) Barris et al. (2004), (8) Riess et al. (2004) 
Table 3. Best fit CMAGIC slopes for well observed high redshift SNe

\begin{tabular}{lrrrrrrr}
\hline \hline IAU Name & \multicolumn{1}{c}{$z$} & $N_{l i n}{ }^{\mathrm{a}}$ & $\chi^{2}$ & DOF $^{\mathrm{b}}$ & $\operatorname{Prob}^{\mathrm{c}}$ & $\beta_{B V}$ & $\sigma_{\beta_{B V}}$ \\
\hline SN1997ce & 0.44 & 4 & 1.41 & 2 & 0.492 & 1.803 & 0.180 \\
SN1997cj & 0.5 & 6 & 1.44 & 4 & 0.839 & 2.159 & 0.264 \\
SN1998aw & 0.44 & 3 & 0.14 & 1 & 0.705 & 2.027 & 0.390 \\
SN1998ax & 0.497 & 3 & 0.43 & 1 & 0.513 & 1.616 & 0.291 \\
SN1998ba & 0.43 & 3 & 0.004 & 1 & 0.952 & 2.222 & 0.359 \\
\hline
\end{tabular}

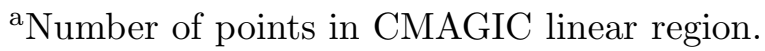

${ }^{\mathrm{b}}$ Degrees of freedom $\left(N_{\text {lin }}-2\right)$ for this fit.

${ }^{c}$ Probability that the $\chi^{2}$ should be worse than the observed value. A high number indicates the fit is 'too good.'

Note. - Results of CMAGIC fits to well observed high redshift SNe. Unlike the fits used in the cosmological analysis, here the stretch $\beta_{B V}$ is fit for each SN.

Table 4. Cuts and parameters for primary fit

\begin{tabular}{lll}
\hline \hline \multicolumn{1}{c}{ Description } & Value & Formal name \\
\hline Minimum redshift cutoff for cosmology fit & 0.01 & zmin \\
Maximum redshift cutoff for cosmology fit & $\mathrm{NA}$ & zmax \\
High redshift cutoff for slope distribution fit & 0.1 & zslopemax \\
Minimum number of points in CMAGIC linear region & 1 & npointsmin \\
Maximum allowable magnitude error & $0.5 \mathrm{mag}$ & magerror \\
Maximum allowable $B-V$ excess at $B_{\text {max }}$ & 0.25 mag & maxcolor \\
Maximum allowable error in date of $B$ maximum & 1.0 days & datemaxerror \\
Minimum stretch allowed & 0.7 & stretchmin \\
Maximum gap between maximum and nearest point in $B$ or $V$ & 7 days & daygap \\
\hline
\end{tabular}

Note. - Cuts and their values for the primary fit. A cut that is not used in the primary fit is given a value of NA. 
Table 5. Identified Systematic Errors

\begin{tabular}{lrrr}
\hline \hline \multicolumn{1}{c}{ Source } & \multicolumn{1}{c}{$\Delta \Omega_{1}{ }^{\mathrm{a}}$} & \multicolumn{1}{c}{$\Delta \Omega_{2}{ }^{\mathrm{b}}$} & \multicolumn{1}{c}{$\Omega_{m}$ (flat) ${ }^{\mathrm{c}}$} \\
\hline Variation of fitting procedures & & & \\
\hline No stretch correction ${ }^{\mathrm{d}}$ & $-0.009(0.07 \sigma)$ & $0.060(0.11 \sigma)$ & $-0.006(0.10 \sigma)$ \\
P99 lightcurve fit & $0.015(0.13 \sigma)$ & $0.144(0.27 \sigma)$ & $-0.007(0.12 \sigma)$ \\
U-enhanced $K$-correction & $-0.052(0.39 \sigma)$ & $0.100(0.19 \sigma)$ & $-0.040(0.70 \sigma)$ \\
Variation of cuts & & & \\
\hline daygap $<5$ & $-0.024(0.18 \sigma)$ & $0.139(0.26 \sigma)$ & $-0.006(0.11 \sigma)$ \\
daygap $<10$ & $0.024(0.20 \sigma)$ & $0.015(0.03 \sigma)$ & $0.014(0.24 \sigma)$ \\
$z>0.015$ & $-0.003(0.02 \sigma)$ & $0.023(0.04 \sigma)$ & $0.00(0.00 \sigma)$ \\
magerror $<0.25$ & $-0.011(0.08 \sigma)$ & $0.015(0.03 \sigma)$ & $-0.006(0.11 \sigma)$ \\
magerror $<1.0$ & $-0.011(0.09 \sigma)$ & $0.017(0.03 \sigma)$ & $-0.006(0.11 \sigma)$ \\
$E(B-V)<0.1$ & $0.018(0.15 \sigma)$ & $-0.467(0.56 \sigma)$ & $0.004(0.07 \sigma)$ \\
$E(B-V)<0.5$ & $-0.048(0.36 \sigma)$ & $-0.00(0.00 \sigma)$ & $0.017(0.29 \sigma)$ \\
datemaxerror $<0.5$ & $0.005(0.04 \sigma)$ & $0.027(0.05 \sigma)$ & $0.00(0.00 \sigma)$ \\
datemaxerror $<2$ & $0.018(0.15 \sigma)$ & $0.115(0.22 \sigma)$ & $0.017(0.29 \sigma)$ \\
npointsmin $>2$ & $0.018(0.15 \sigma)$ & $0.229(0.43 \sigma)$ & $-0.006(0.11 \sigma)$ \\
Other systematics & & & \\
\hline Hamuy, Riess, Jha only & $-0.015(0.11 \sigma)$ & $-0.037(0.04 \sigma)$ & $-0.006(0.11 \sigma)$ \\
Jack-Knife: SN2001ix & $-0.014(0.11 \sigma)$ & $-0.281(0.33 \sigma)$ & $-0.013(0.23 \sigma)$ \\
Jack-Knife: SN2002kd & $0.015(0.12 \sigma)$ & $0.310(0.58 \sigma)$ & $-0.016(0.28 \sigma)$ \\
$\sigma_{\text {int }}=0.08$ & $-0.015(0.11 \sigma)$ & $0.022(0.04 \sigma)$ & $-0.013(0.23 \sigma)$ \\
$\sigma_{\text {int }}=0.15$ & $0.010(0.09 \sigma)$ & $-0.015(0.02 \sigma)$ & $0.006(0.11 \sigma)$ \\
Malmquist bias & $-0.0012(0.01 \sigma)$ & $0.045(0.08 \sigma)$ & $-0.003(0.05 \sigma)$ \\
Bumps & $0.005(0.04 \sigma)$ & $-0.014(0.017)$ & $0.003(0.05 \sigma)$ \\
\hline & & & \\
\hline & & & \\
& & & \\
& & & \\
& & &
\end{tabular}

${ }^{a}$ Shift in $\Omega_{1} \equiv 0.790 \Omega_{m}-0.613 \Omega_{\Lambda}$ (the short axis).

${ }^{\mathrm{b}}$ Shift in $\Omega_{2} \equiv 0.613 \Omega_{m}+0.790 \Omega_{\Lambda}$ (the long axis).

${ }^{\mathrm{c}}$ Shift in the value of $\Omega_{m}$ assuming a flat universe $\left(\Omega_{m}+\Omega_{\Lambda}=1\right)$

${ }^{\mathrm{d}}$ Not used in final determination of systematic errors.

Note. - Identified systematic errors, as detailed in 8 The names of the cuts referenced above are as defined in table 4 


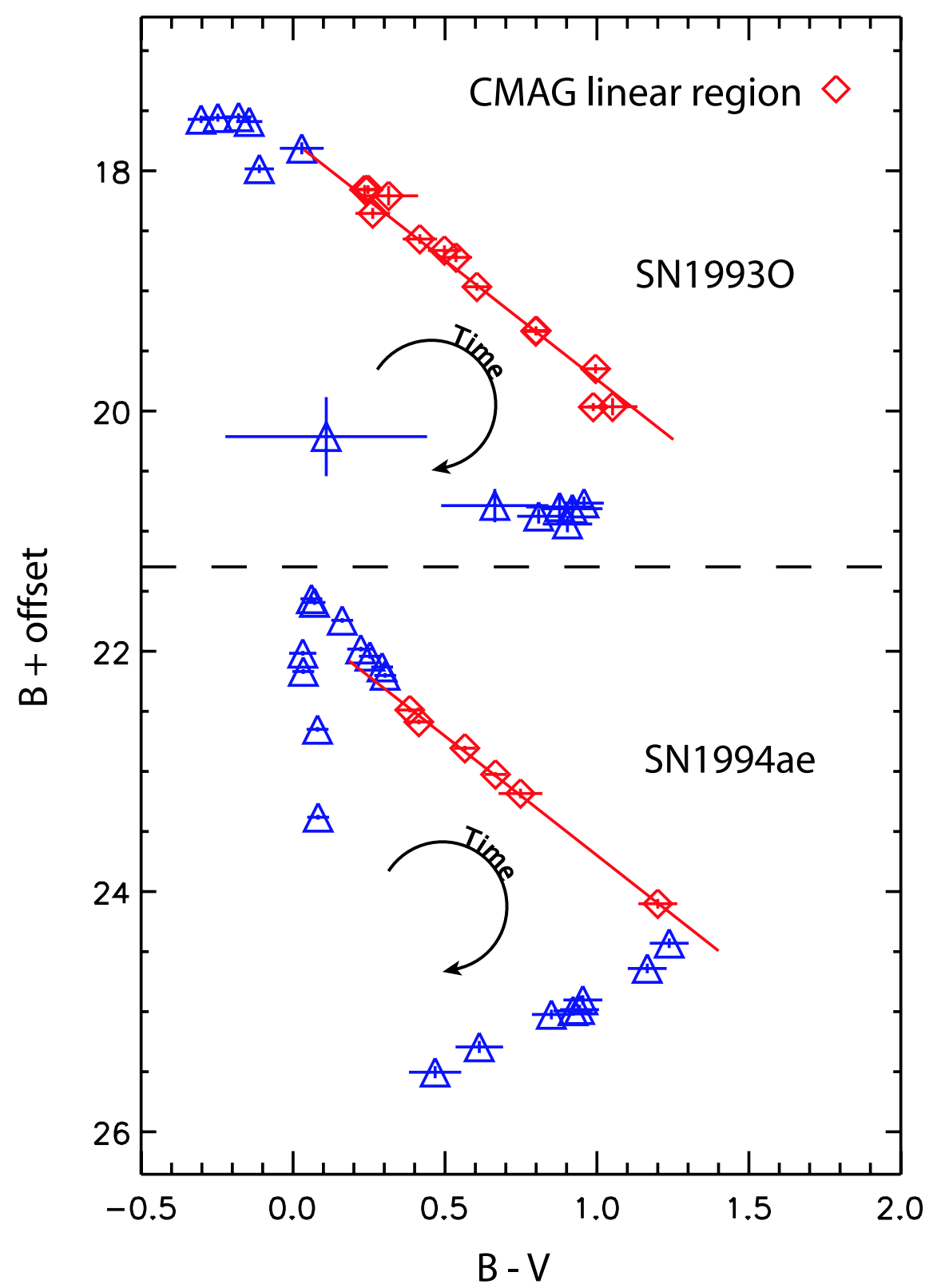

Fig. 1. $-B$ vs. $B-V$ CMAGIC diagrams for SN1993O, a fairly typical low redshift SN Ia (z= 0.052 , stretch $=0.927)$, and SN1994ae $(\mathrm{z}=0.004$, stretch $=1.006)$. The points in the linear region (based on the date relative to maximum light) are shown as diamonds for both SNe. The slopes were fixed at $\beta_{B V}=1.98$ for this fit. SN1994ae displays a bump feature prior to the linear region. 


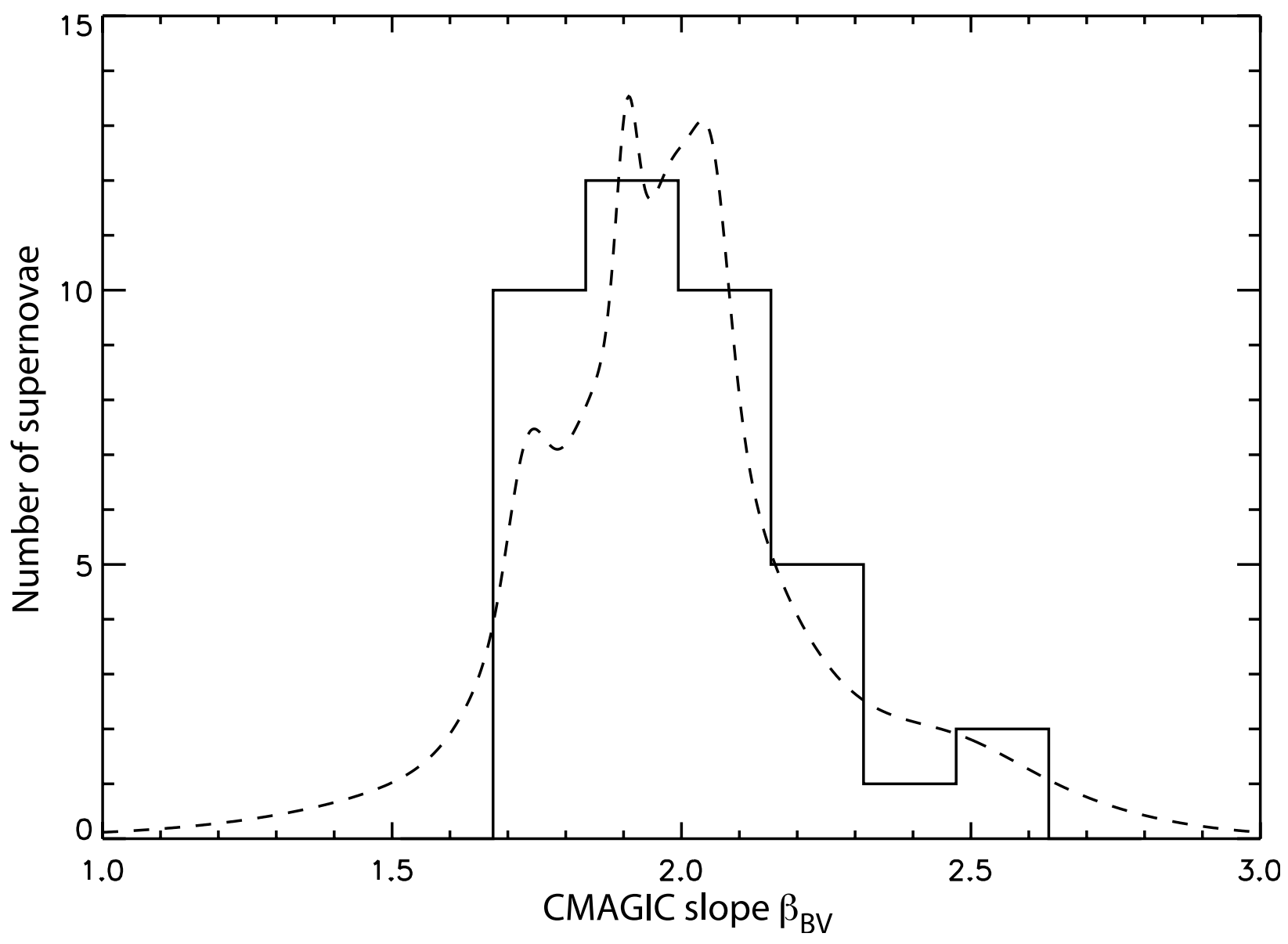

Fig. 2.- Histogram of $\beta_{B V}$ for the 44 low redshift SNe Ia used in the primary fit, after $K$-correction (solid line). Overlain is an ideogram of the same distribution (dashed line). The ideogram is constructed by adding a Gaussian of the appropriate width and mean value for each SN, representing the best fit value of $\beta_{B V}$ and its associated measurement error of each object. This shows how the size of the measurement errors are affecting the distribution. The mean of this distribution is 1.99 and the RMS is 0.16 . The binning is purely for display purposes. 


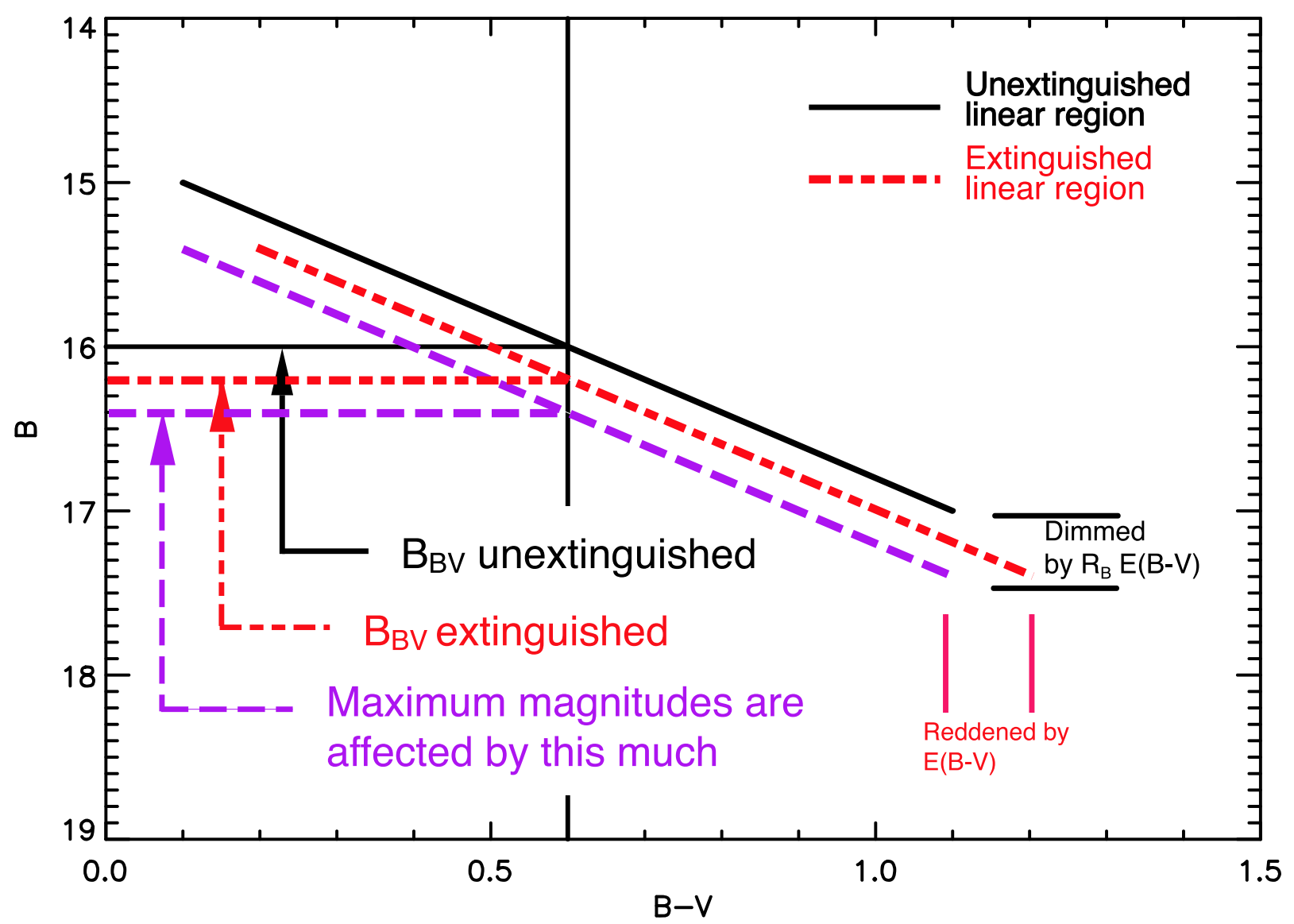

Fig. 3.- Schematic representation of how host galaxy dust affects $B_{B V 0.6}$. Ordinary dust both extinguishes and reddens light. Here an extinction of $A_{B}=0.4 \mathrm{mag}$ is shown. The solid line represents the unextinguished linear region. The bottom line represents the effects of extinction without reddening, the middle line includes both the dimming and reddening effects. The critical point is that, because the linear relation is always evaluated at the same color to form $B_{B V 0.6}$, the two effects partially cancel. 


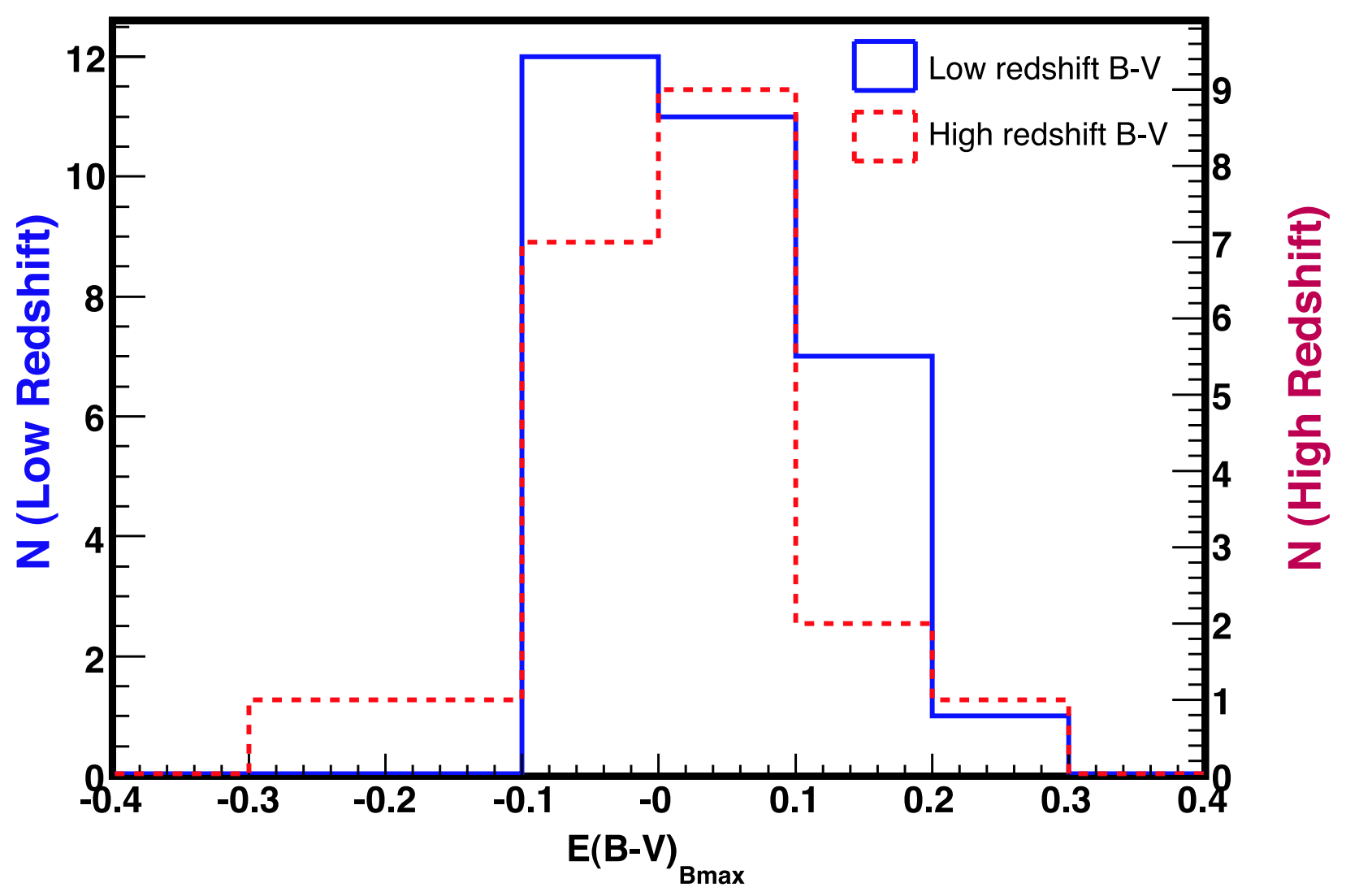

Fig. 4.- Histogram of $E(B-V)_{B \max }$ at $B$ maximum for the low and high redshift primary fit sample. The high redshift histogram (dashed line,scale on right) has been scaled to the low redshift histogram (solid line, scale on left) for display purposes. The mean color of the low redshift sample is $0.045 \pm 0.027$ and that of the high redshift sample is $0.027 \pm 0.019$. The two distributions are consistent given the small number of events in each bin. 


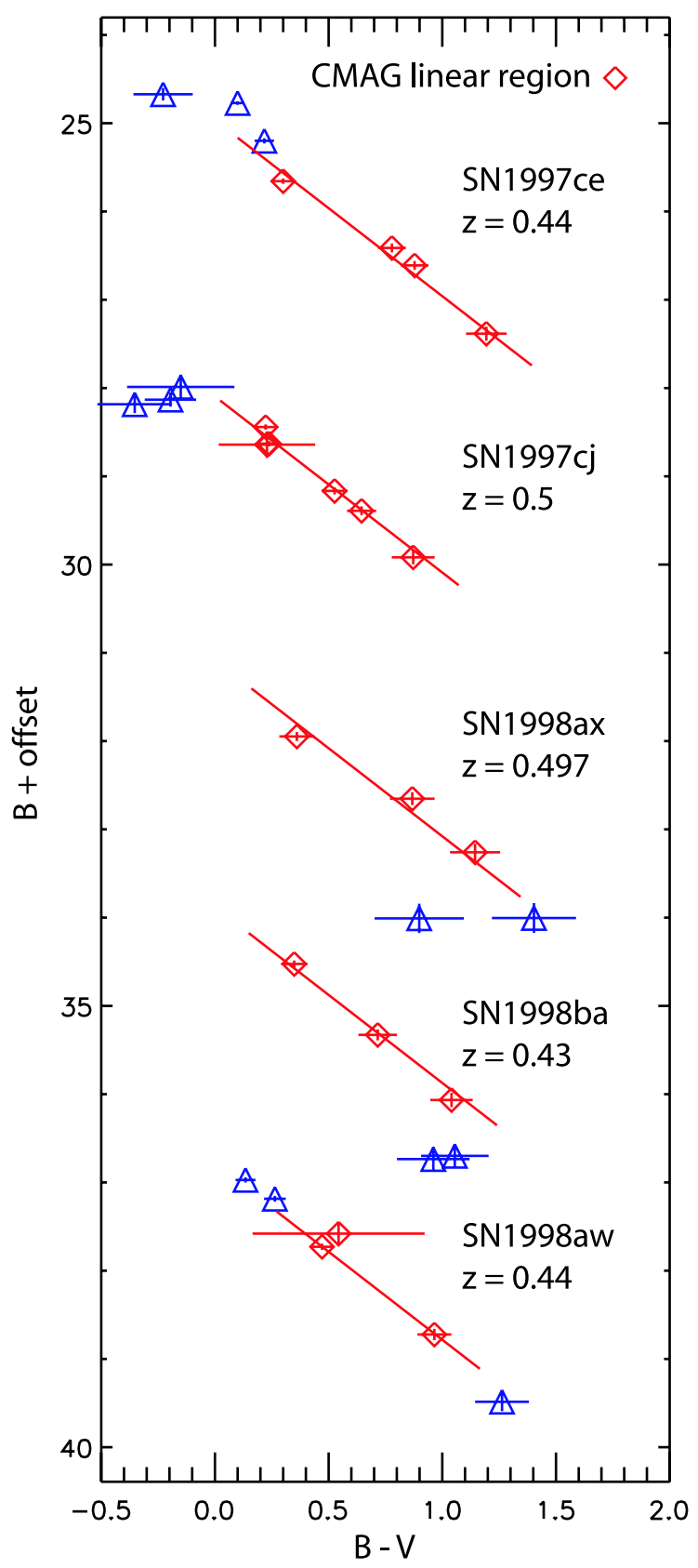

Fig. 5. - CMAGIC diagrams for well observed high redshift SNe Ia: SN1997ce (z=0.44), SN1997cj $(z=0.5)$, SN1998ax $(z=0.497)$, SN1998ba $(z=0.43)$ and SN1998aw $(z=0.44)$. Note the prominent bump before the linear region for SN1997ce. The points in the linear regions are shown as diamonds. 


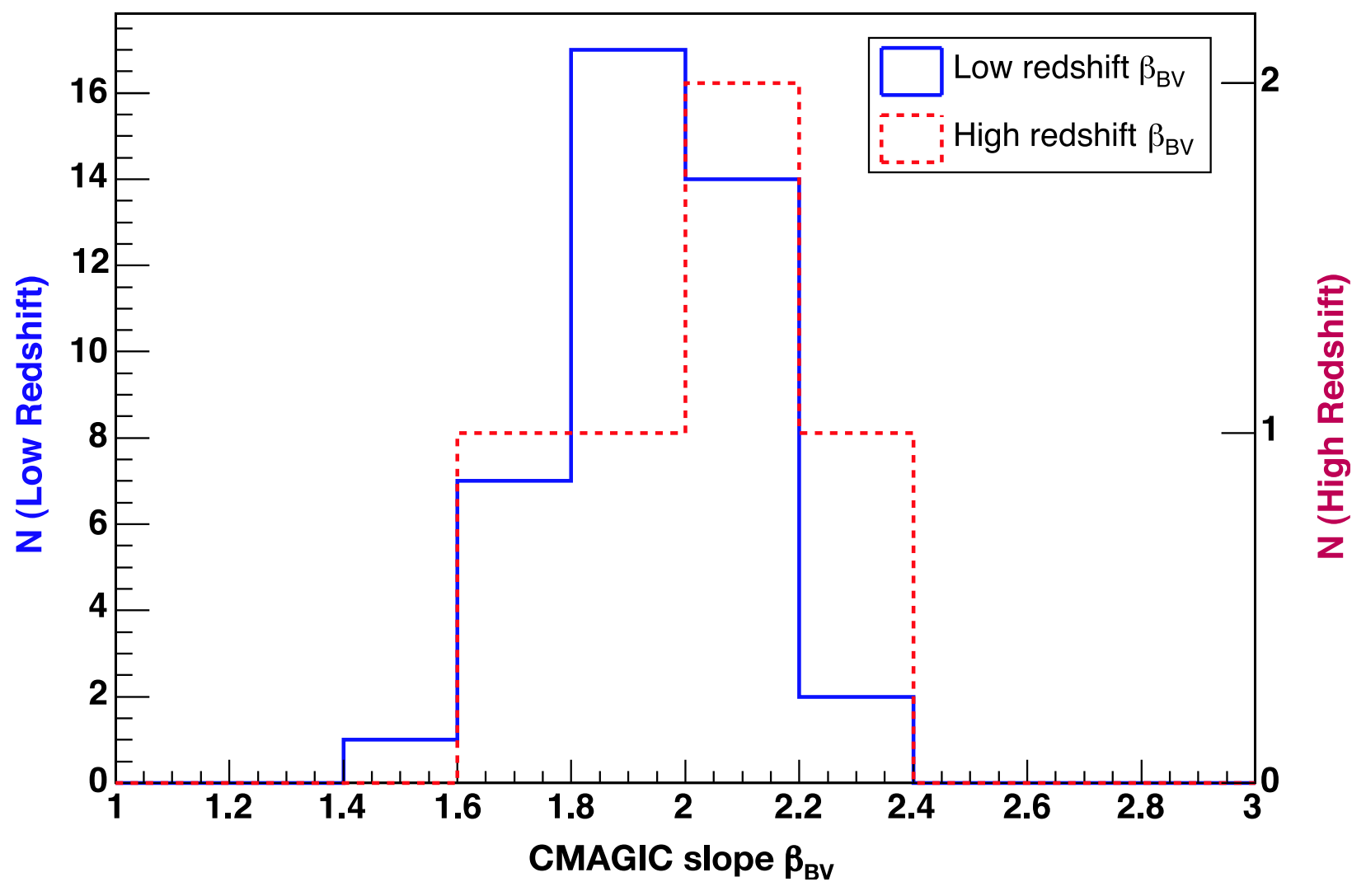

Fig. 6. - Histogram of CMAGIC slopes for low and high redshift primary fit samples. The high redshift histogram (scale on the right) has been scaled to the low redshift histogram (scale on the left) for display purposes. 


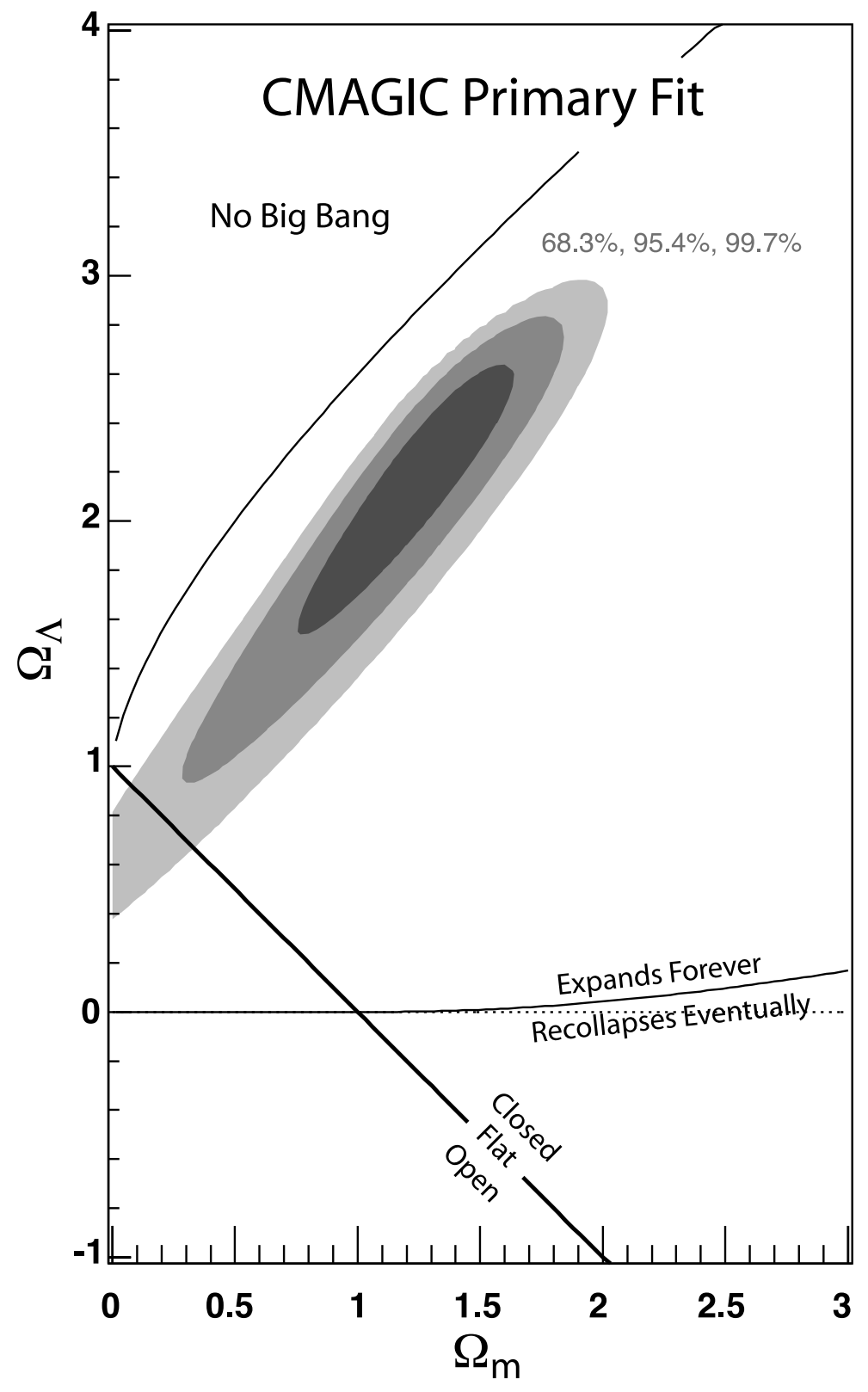

Fig. 7.- Confidence regions for $\Omega_{m}, \Omega_{\Lambda}$ from the primary fit. The contours represent $68.3 \%$, $95.4 \%$, and $99.7 \%$ of the total probability. 


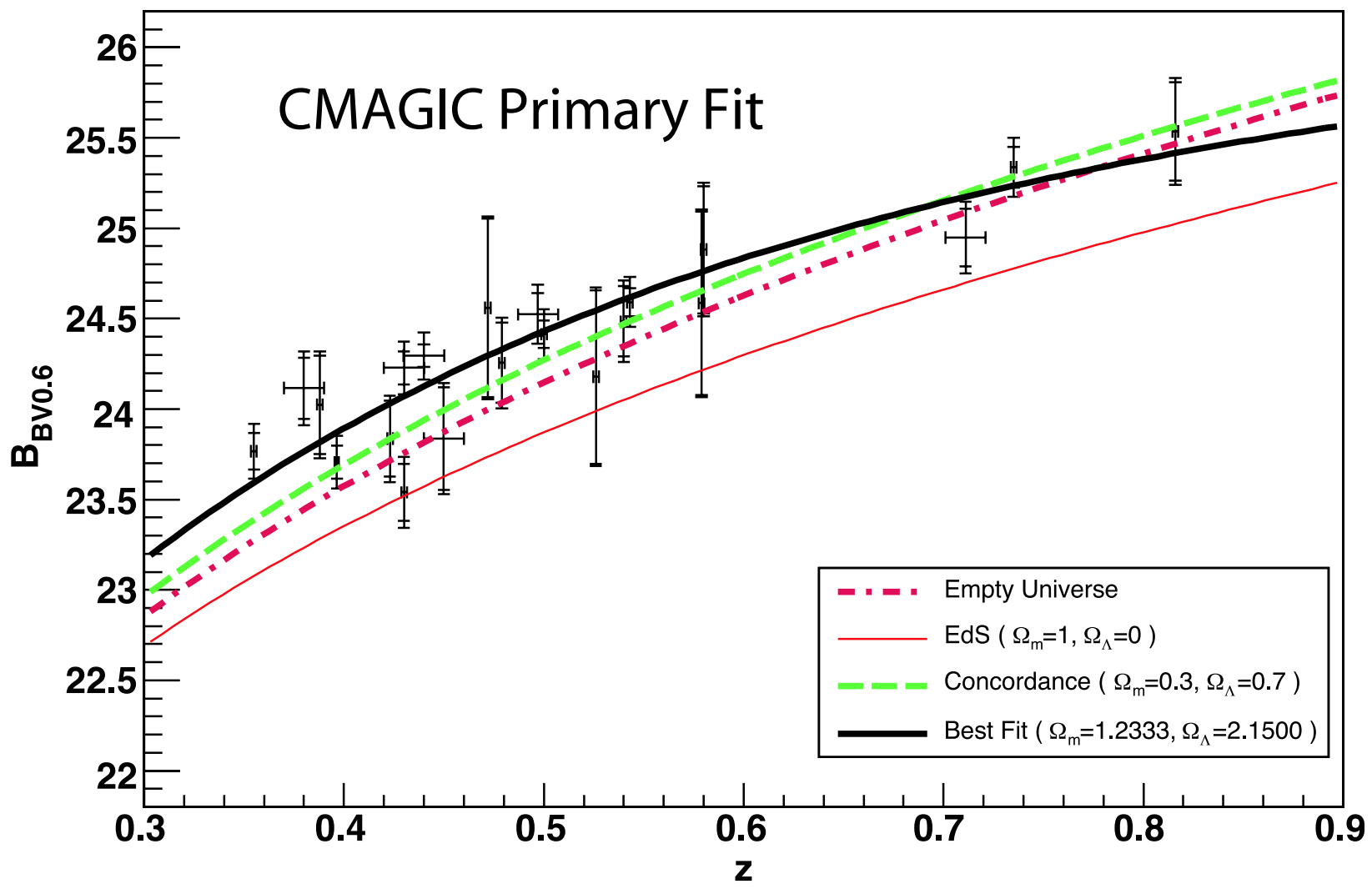

Fig. 8. - Hubble diagram for the primary fit. The outer error bars include both $\sigma_{\text {int }}=0.12$ of intrinsic scatter derived from low redshift fits and the contribution of the peculiar velocity errors (which are also shown as the horizontal error bars); the inner ones do not. Also shown are the relations for an empty Universe, an Einstein-deSitter one, and the "concordance" cosmology with $\Omega_{m}=0.3, \Omega_{\Lambda}=0.7$. 


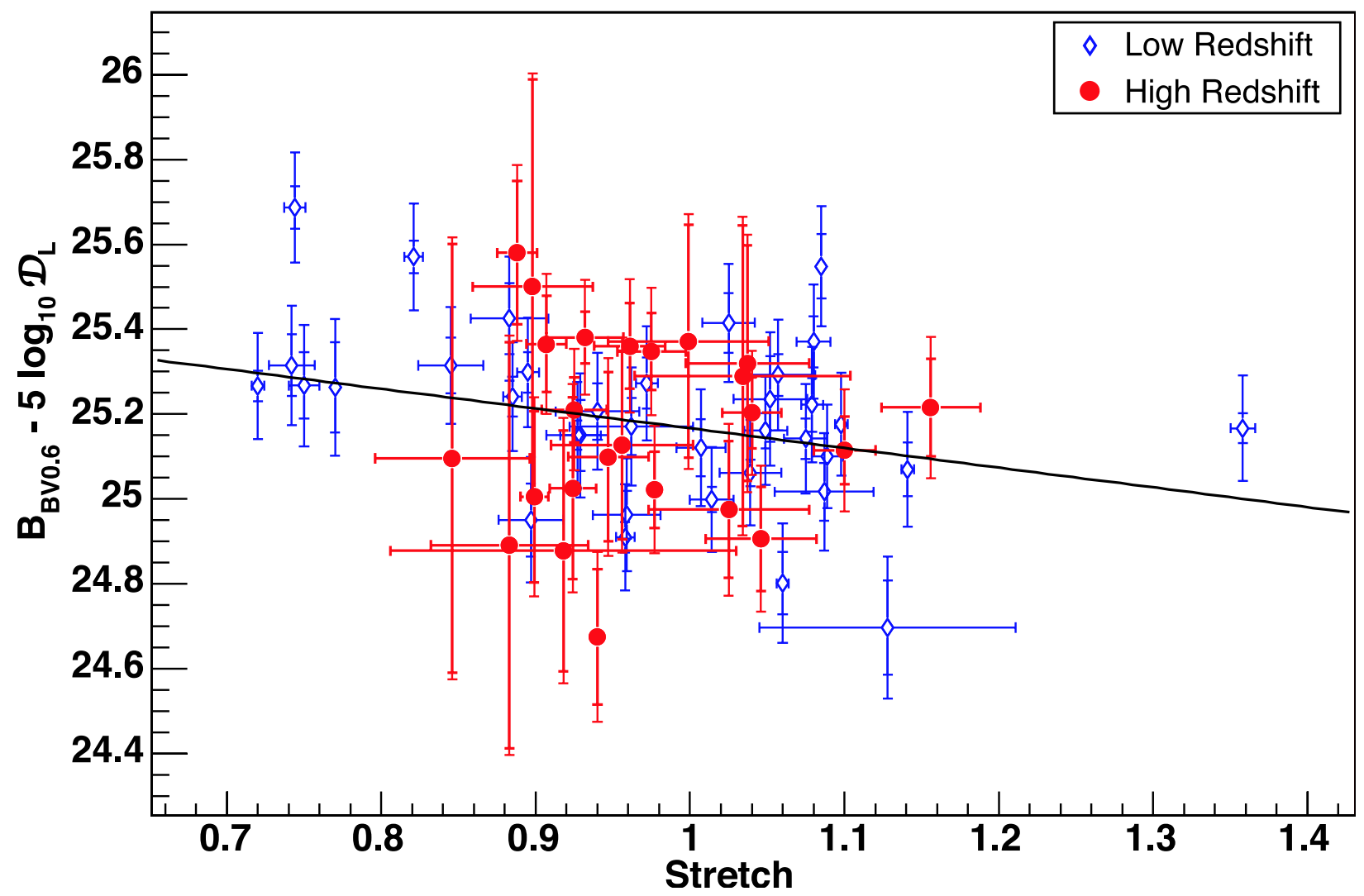

Fig. 9.- Stretch-luminosity relationship for the low redshift SNe (open diamonds) and high redshift SNe (filled circles). Each point is $B_{B V 0.6}$ minus the $c / H_{0}$ free luminosity distance of equation 3 , plotted against the stretch of the SN. The line represents the fit estimates for $\alpha$ and $\mathcal{M}$ from the primary fit $(\alpha=0.52, \mathcal{M}=25.17)$. The outer error bars include 0.12 magnitudes of intrinsic scatter. 


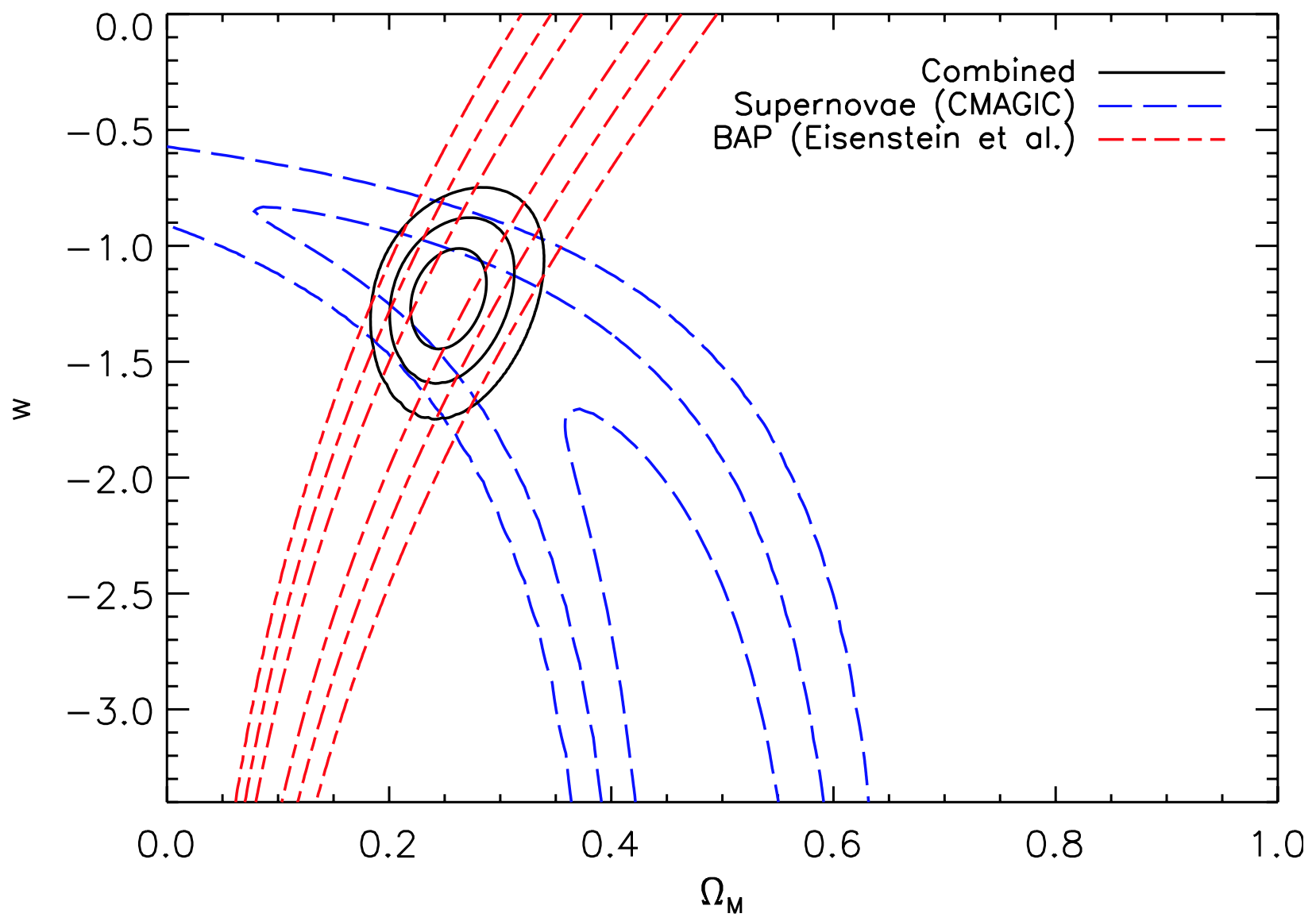

Fig. 10.- Confidence regions for $\mathrm{w}, \Omega_{m}$ assuming a flat universe. The contours represent $68.3 \%$, $95.4 \%$, and $99.7 \%$ of the total probability. Both the constraints from the CMAGIC analysis of supernovae and the baryon acoustic peak (BAP) of Eisenstein et al. (2005) are shown, as are the contours that result from combining the two measurements. A cosmological constant corresponds to $w=-1$. 


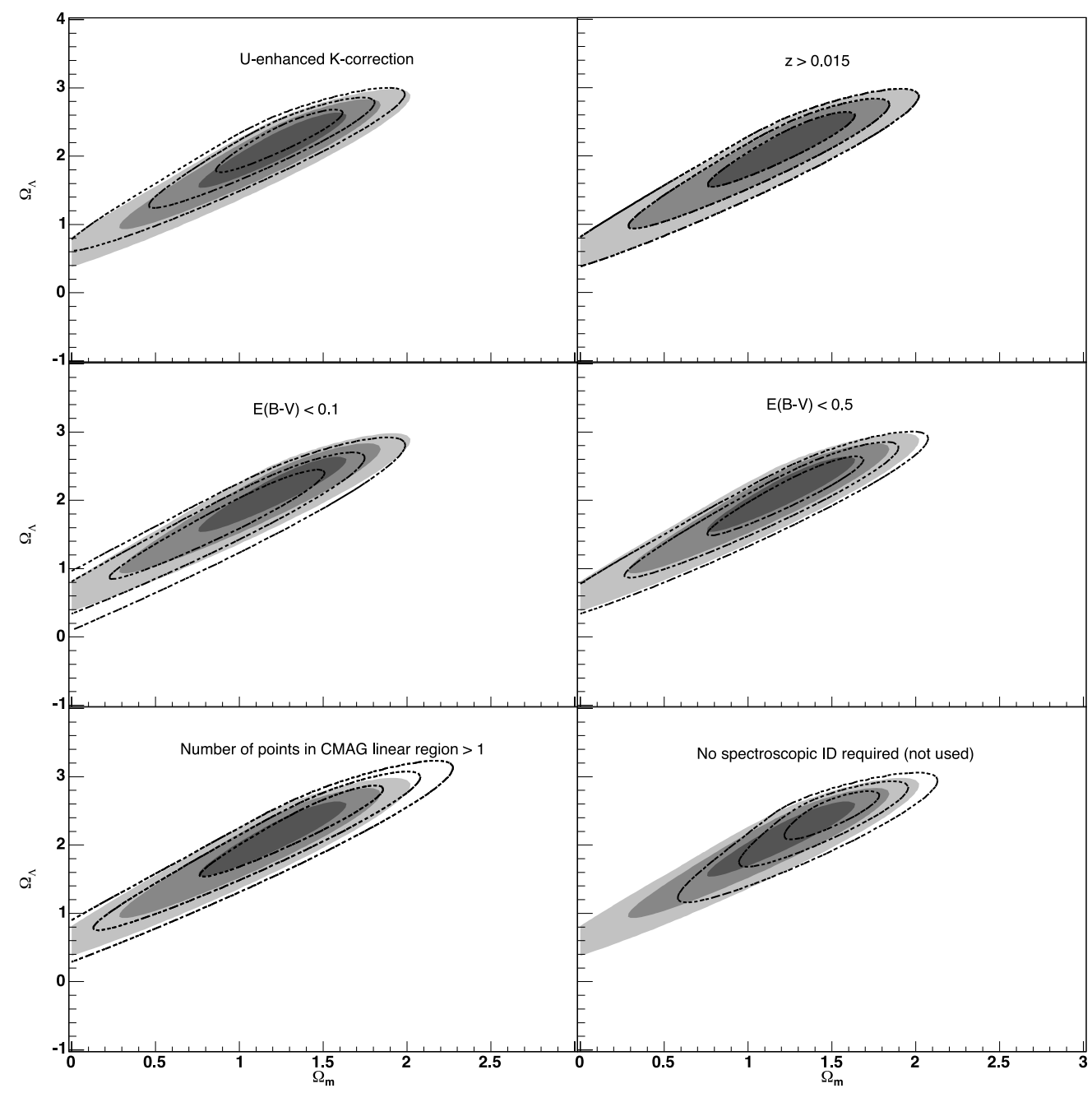

Fig. 11. - Examples of some of the systematics considered in this analysis. More complete descriptions can be found in the text. In each panel, the primary fit is shown as filled contours and the fit with the specified change is shown as dashed contours. For both sets the contours correspond to $68.3 \%, 95.4 \%$, and $99.7 \%$ of the total probability. Note that the scenario represented in the bottom right panel (not requiring spectroscopic ID) is not used in the final systematics estimate, as this is considered unmotivated. 


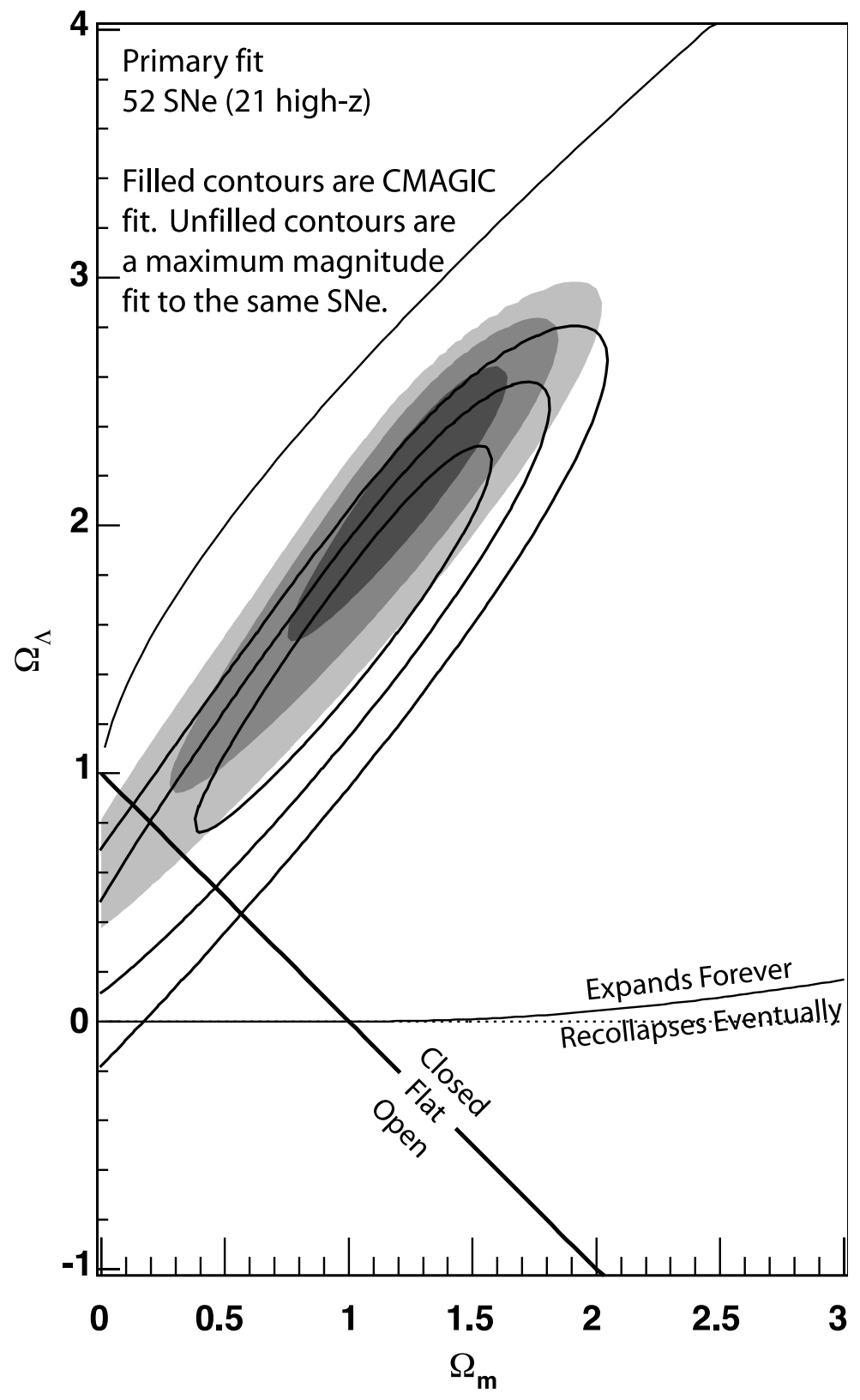

Fig. 12.- Direct comparison of the $m_{B}$ and $B_{B V 0.6}$ cosmology results. The $m_{B}$ results are shown as unfilled contours and the $B_{B V 0.6}$ results as filled ones. In both cases, the contours represent $68.3 \%, 95.4 \%$, and $99.7 \%$ of the total probability. 


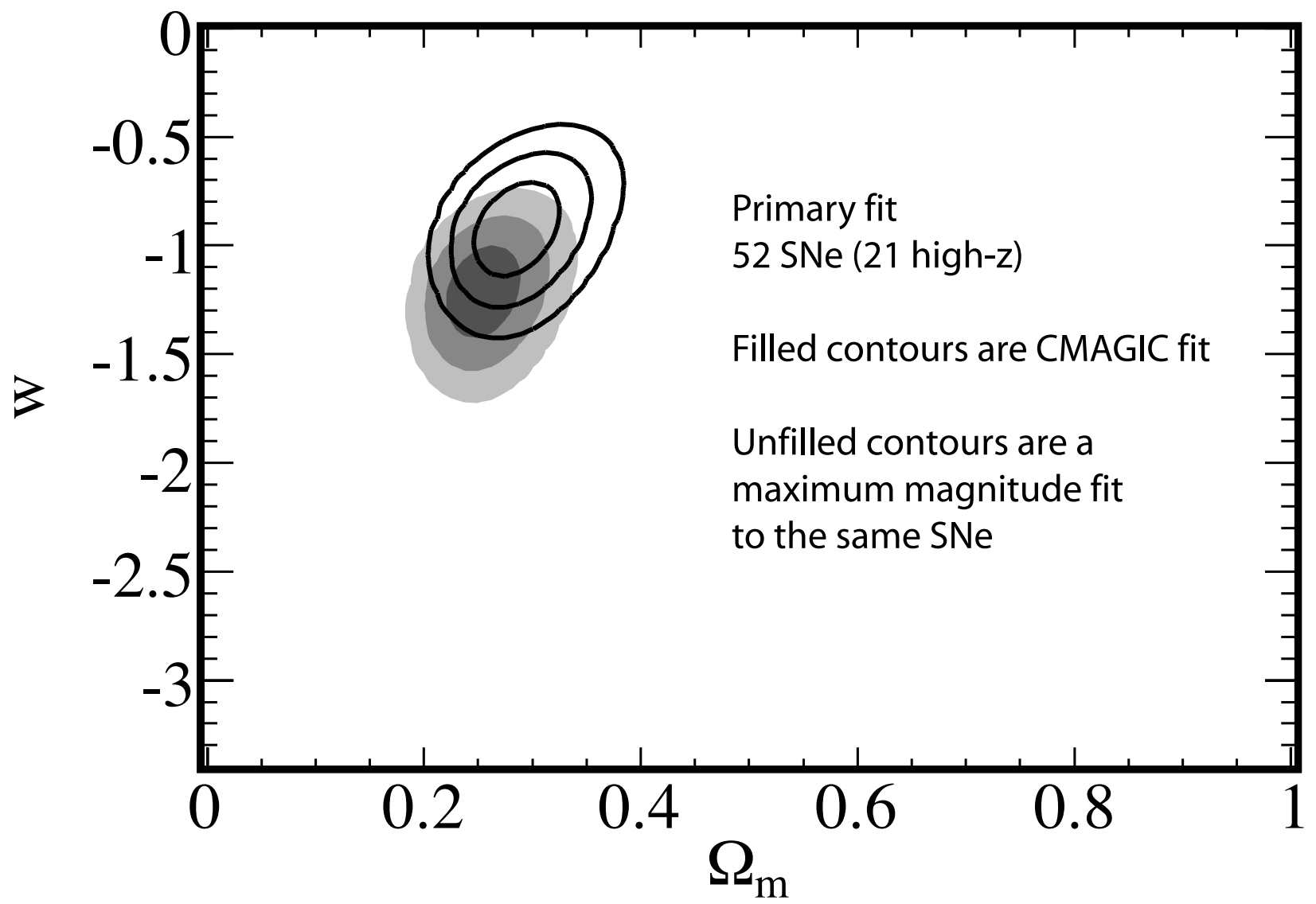

Fig. 13. - Direct comparison of the $m_{B}$ and $B_{B V 0.6}$ cosmology fits to $w, \Omega_{m}$, assuming a flat universe and including the baryon acoustic peak measurement of Eisenstein et al. (2005). The $m_{B}$ results are shown as unfilled contours and the $B_{B V 0.6}$ results as filled ones. In both cases, the contours represent $68.3 \%, 95.4 \%$, and $99.7 \%$ of the total probability. Note that the difference between the two results is certainly not independent of the difference between the $m_{B}$ and $B_{B V 0.6}$ results for $\Omega_{m}$ and $\Omega_{\Lambda}$. 


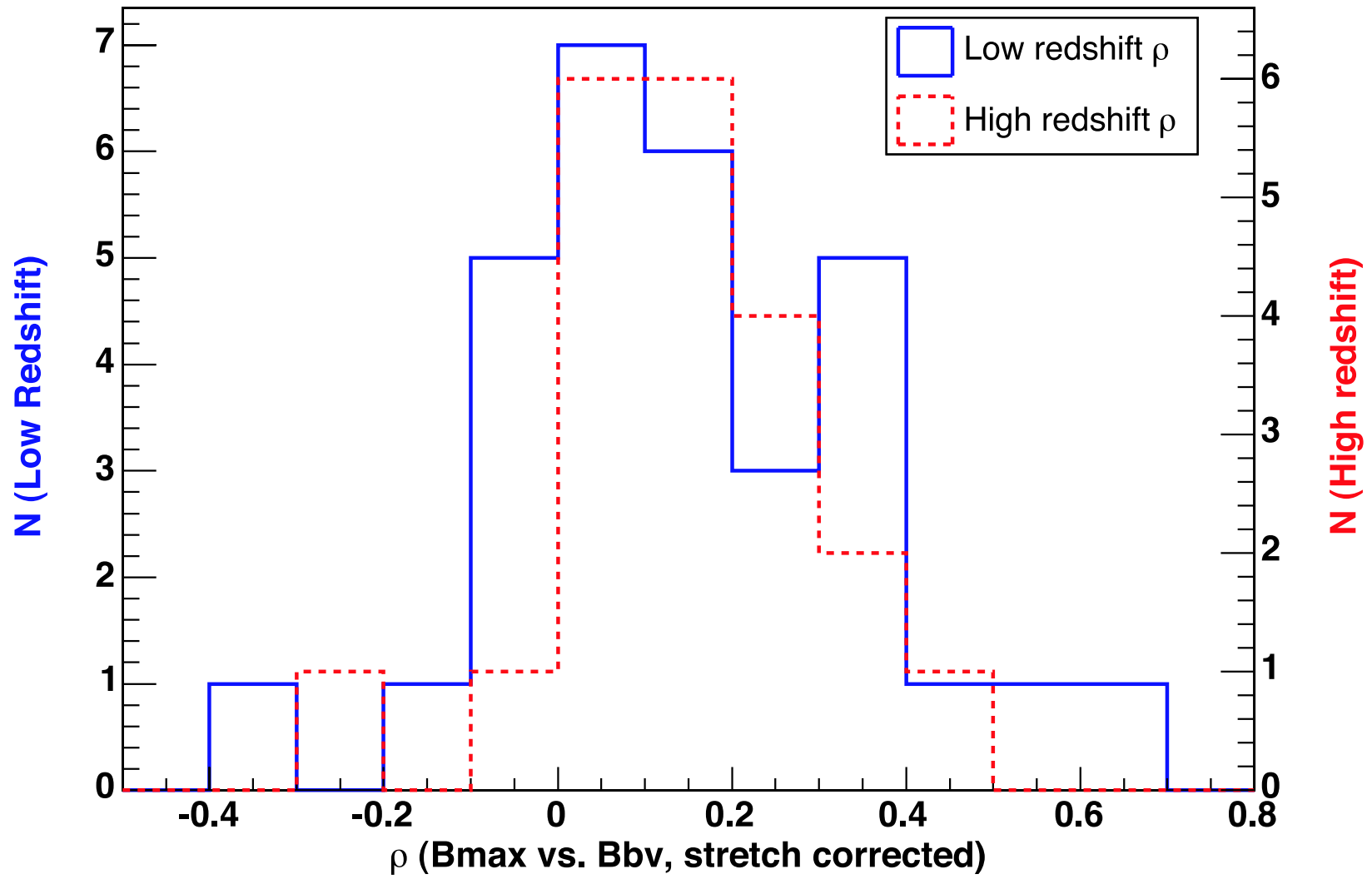

Fig. 14. - The histogram of correlation coefficients $(\rho)$ for $m_{B}, B_{B V 0.6}$ after stretch correction. This only includes the correlations induced by the fitting, and does not include any due to the intrinsic variability of SNe Ia, or that which arises from peculiar velocity errors. 


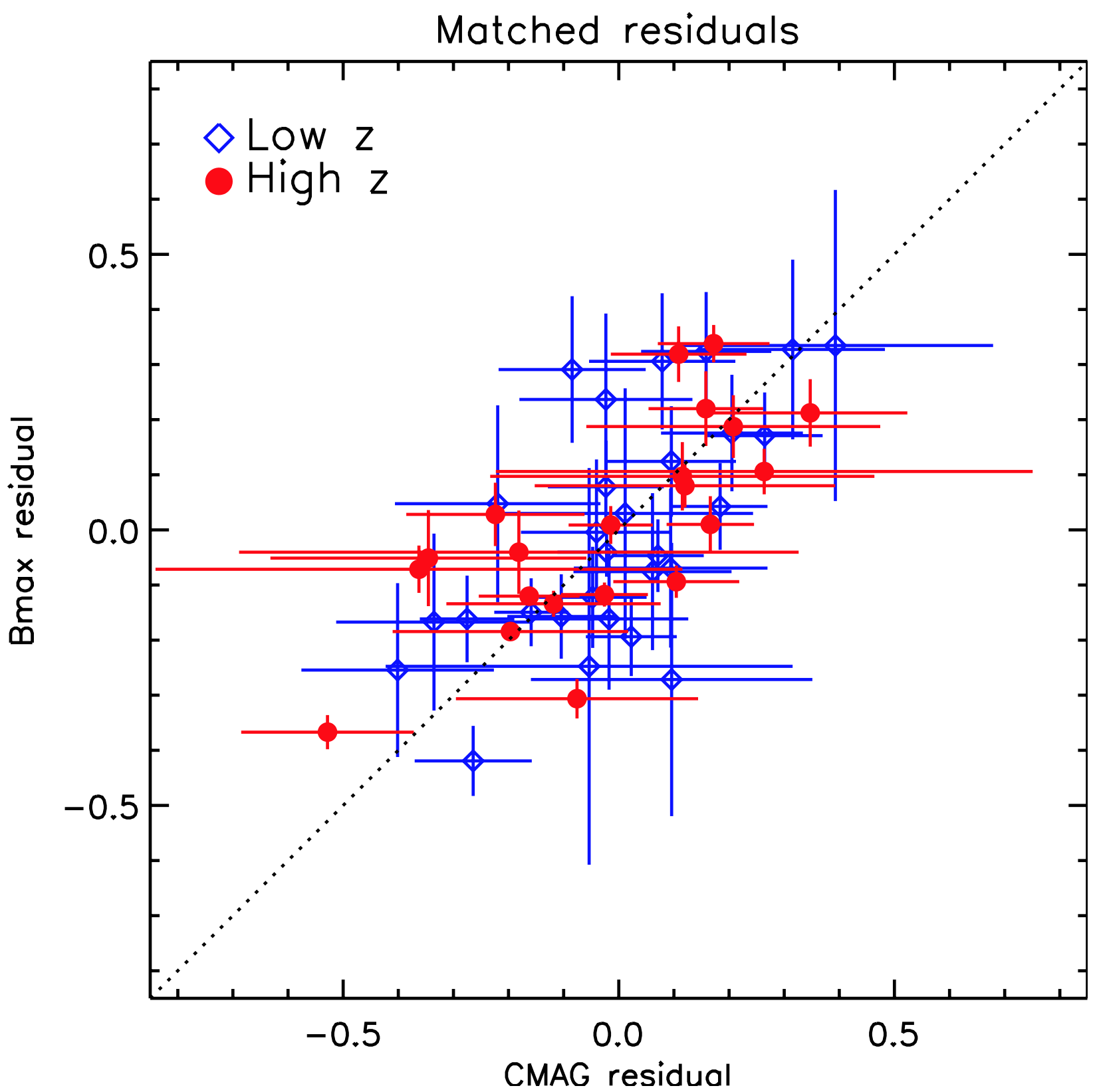

Fig. 15.- Residuals from Hubble line for the $m_{B}$ fit and the $B_{B V 0.6}$ fit. Overlain is a line with slope 1. The high redshift data is shown as filled circles and the low redshift data as open diamonds. The correlation of the low redshift sample is mostly explained by the effects of peculiar velocities. 